\title{
Searching for THz Gunn oscillations in GaN planar nanodiodes
}

\author{
A. Íñiguez-de-la-Torre, I. Íñiguez-de-la-Torre, J. Mateos, and T. González \\ Departamento de Física Aplicada, Universidad de Salamanca, Plaza de la Merced s/n, 37008 \\ Salamanca, Spain
}

\author{
P. Sangaré, M. Faucher, B. Grimbert, V. Brandli, G. Ducournau, and C. Gaquière \\ Institut d'Electronique de Microélectronique et de Nanotechnologie (IEMN), UMR CNRS 8520, \\ Université de Lille 1, Avenue Poincaré BP60069, 59652, Villeneuve d'Ascq CEDEX, France
}

\begin{abstract}
A detailed study of GaN-based planar asymmetric nanodiodes, promising devices for the fabrication of room temperature $\mathrm{THz}$ Gunn oscillators, is reported. By using Monte Carlo simulations, an analysis of the static $I-V$ curves and the time-domain evolution of the current obtained when varying some simulation parameters in the diodes has been made. Oscillation frequencies of hundreds of $\mathrm{GHz}$ are predicted by the simulations in diodes with micrometric channel lengths. Following simulation guidelines, a first batch of diodes was fabricated. It was found that surface charge depletion effects are stronger than expected and inhibit the onset of the oscillations. Indeed, a simple standard constant surface charge model is not able to reproduce experimental measurements and a self-consistent model must be included in the simulations. Using a self-consistent model it was found that to achieve oscillations wider channels and improved geometries are necessary.
\end{abstract}




\section{INTRODUCTION}

Until the last few years the terahertz frequency range, sandwiched between the far infrared and the millimetre waves, was one of the least explored regions of the electromagnetic spectrum, but nowadays there are many efforts trying to conquer the "Terahertz gap" because of its countless applications in a wide range of fields $[1,2]$. Nevertheless, there is still a long way to go in order to efficiently achieve such an ambitious goal, mainly due to the lack of room temperature $\mathrm{THz}$ sources. One possibility to follow up is to take advantage from well known phenomena and devices usually employed in the microwave field and try to adapt them for higher frequency operation. In this line, we propose to exploit Gunn oscillations in GaN-based nanodiodes to obtain room temperature sources.

Microwave radiation sources based on Gunn effect [3] have been developed for almost 50 years. Currently the key aspect is to improve power and heat dissipation, and to increase the oscillation frequency of these Gunn oscillators. GaN has several features that turn this semiconductor into a promising candidate in this field. We are referring to its high electrical strength, directly related to its large energy gap, as well as its high peak and saturation velocity and its low relaxation time as compared with other III-V materials typically used in Gunn diodes [4]. In order to improve the heat dissipation and design flexibility with respect to the vertical structures previously explored (with little success to obtain Gunn oscillations in GaN diodes) [57], we have chosen a planar topology as those proposed for GaAs/AlGaAs and InGaAs/AlGaAs diodes in $[8,9]$ but using $\mathrm{GaN}$ to take advantage of its excellent properties. In this paper we will investigate the use of the special geometry of planar asymmetric nanodiodes, so-called selfswitching diodes (SSDs), to provide Gunn oscillations. These diodes were proposed by A. M. Song et al. in 2003 [10], and since then this seemingly simple device has already demonstrated detection capability up to $110 \mathrm{GHz}$ in a guided setup [11], and up to $1.5 \mathrm{THz}$ in a free space system [12], both at room temperature, and even up to $2.5 \mathrm{THz}$ at $10 \mathrm{~K}$ in free space [13]. The challenge now is to use it as emitter.

In previous works, by means of Monte Carlo (MC) simulations [14], the physics of the operation of SSDs and their static [15], dynamic $[16,17]$ and noise properties $[18,19]$ have been successfully explained. Our research focus, motivated by the work presented in [20], showing that the appearance of Gunn oscillations is promoted by the shape of the SSDs, is the design and fabrication of a compact, cheap and room temperature Gunn source approaching the $\mathrm{THz}$ range using GaN-based SSDs. The initial numerical estimations predict high frequency oscillations at hundreds of $\mathrm{GHz}[21,22]$, but no experimental evidence has been reported yet. Therefore, the 
aim of this work is to improve the modeling and to study in detail the possibility of achieving $\mathrm{THz}$ oscillations by means of Gunn effect in GaN SSDs. To this end, MC simulations of the diodes were initially performed. Then, a first batch of GaN SSDs was fabricated, the results of their experimental characterization being compared with simulations.

The content of this paper is structured as follows. In Sec. II the numerical model and the main features of the simulated structures are explained. Section III presents the influence on Gunn oscillations of some characteristic parameters of the SSDs (both geometrical and of the MC model) obtained with a simple standard constant surface charge model. In Sec. IV we carry out a comparison of those preliminary results with the experimental ones from a first fabrication run, showing the need for an improved self-consistent surface charge model, taking into account a more realistic computation of the surface charge value. Also, the design strategies to experimentally attain $\mathrm{THz}$ generation are presented. Finally, in Sec. IV, the main conclusions of this work are drawn.

\section{MONTE CARLO MODEL AND DIODES UNDER STUDY}

To model SSDs, whose typical geometry is shown in Fig. 1(a), we have made use of an in-house 2D simulator that consists of a semiclassical ensemble MC code self-consistently coupled with a 2D Poisson solver. The conduction band of GaN (Wurtzite crystal structure) is modeled by three non-parabolic spherical valleys $\left(\Gamma_{1}, U\right.$ and $\left.\Gamma_{3}\right)$, and the considered scattering mechanisms are acoustic, optical and intervalley phonons, ionized impurities and piezoelectric scattering. Obviously, because of the geometry of the devices, a 3D simulator would have been more appropriate, but it would add a huge computational effort and contribute with little new information [23]. So, as shown in Fig. 1(b), where the shape of the simulation domain (which includes access regions) is sketched, only the GaN layer is considered in the so-called "topview" simulations, where we include two ad hoc parameters: i) a "virtual" background net doping, $N_{\mathrm{Db}}$, is assigned to this layer (although the associated scattering is not considered) in order to account for the fixed charges at the top surface and the heterojunction, and ii) a negative surface charge density, $\sigma$, is assigned to the semiconductor-air interfaces to account for the influence of the surface states originated during the etching process. $N_{\mathrm{Db}}$ and $\sigma$ are the only two parameters of our MC model to be fixed by comparison of simulations with experiments. The DC voltages are applied by step-like biasing. More information about the simulator can be found in $[24,25]$. 
The negative value of the surface charge $\sigma$ (associated with electrons trapped at surface states) induces that, as a consequence of Coulomb repulsion, the carrier density near the sidewalls is lowered, thus leading to a depletion of the channel. The depletion width at each side of the channel can be estimated as $W_{\mathrm{d}}=\sigma / N_{\mathrm{Db}}$. Therefore, the effective conduction width is $W_{\text {eff }}=W-2 W_{\mathrm{d}}$, with $W$ the lithographic width of the channel. The simplest and more standard model for $\sigma$ (constant surface charge model), is to give it a constant value independently of the topology, bias and time. This model is valid under certain conditions: not very small devices (larger than the depletion width) and low bias. In order to correctly simulate the global effect of surface charges on the electron transport in nanodevices we have developed a self-consistent surface charge model of further validity. In such an improved model the local value of the surface charge is continuously updated according to the value of the carrier density nearby until a stationary behavior is achieved. Further details of this model can be found in [26, 27].

The DC to AC conversion efficiency is evaluated in order to analyze the SSDs capability as emitters. To this end, we used a common technique already used by other authors [28-30]: a single-tone sinusoidal potential of amplitude $V_{\mathrm{AC}}$ is superimposed to a DC bias $V_{\mathrm{DC}}$. These working conditions are somehow equivalent to the operation of the diode in a resonant circuit with appropriate elements, as shown in [30]. Under such conditions, the dissipated DC power, $P_{\mathrm{DC}}$, and the time-average $\mathrm{AC}$ power, $P_{\mathrm{AC}}$, are evaluated and the conversion efficiency, $\eta$, is calculated as the ratio between $P_{\mathrm{AC}}$ and $P_{\mathrm{DC}}$, with a minus sign: $\eta=-P_{\mathrm{AC}} / P_{\mathrm{DC}}$. Therefore, negative values of $\eta$ indicate a resistive behavior of the diode, while positive values mean AC generation from DC. Also, in order to perform a spectral analysis of the current oscillations (when $\eta$ is positive), the $\mathrm{MC}$ extracted current sequences, $I(t)$, are transformed into the frequency domain by means of fast Fourier transform (FFT) to determine its spectral density, $S_{I}(f)$. We will present both the values of $S_{I}(f)$ and $I$ normalized in such a way that their absolute values (in $\mathrm{A}^{2} \mathrm{~s}$ for $S_{I}$ and A for $I$ ) in a given device can be obtained just multiplying by the experimental sheet electron density in the channel (in units of $\mathrm{m}^{-2}$ ).

\section{MONTE CARLO WITH CONSTANT SURFACE CHARGE MODEL: ANALYSIS OF BASIC DEPENDENCES}

Previously to the fabrication of the GaN SSDs we have performed simulations of different devices using the simple (and fast) constant surface charge model in order to study the ability of these nanodevices to generate Gunn oscillations at room temperature. We have used this simple

model to analyze the influence of some characteristic parameters (both geometrical and of the 
MC model), in order to identify the individual influence of each of them avoiding the complex effects arising when the surface charges are not fixed. We will consider as reference structure a diode with $L=1 \mu \mathrm{m}, W=150 \mathrm{~nm}, W_{\mathrm{V}}=200 \mathrm{~nm}, W_{\mathrm{H}}=100 \mathrm{~nm}, C=600 \mathrm{~nm}$, with a background doping $N_{\mathrm{Db}}=2 \times 10^{17} \mathrm{~cm}^{-3}$ and a surface charge density $\sigma=-0.75 \times 10^{12} \mathrm{~cm}^{-2}$. As a general feature, this structure shows oscillations in the current when the applied voltage exceeds $30 \mathrm{~V}$, originated by the classical Gunn effect, but no evidence of the onset of the oscillations is observed in the $I-V$ curves as shown in Figs. 2 and 3 (the values of the current are obtained as the time average over long simulations, including a large number of periods when oscillations are present).

As the two critical parameters in our model are $\sigma$ and $N_{\mathrm{Db}}$ (whose values are unknown and should be determined by comparison of simulated results with measurements), the first set of simulations, Fig. 2, analyzes the influence of these parameters. The $I-V$ curves of the reference structure and time sequences of the current for an applied voltage of $50 \mathrm{~V}$, for which this diode exhibits small-amplitude Gunn oscillations (around $300 \mathrm{GHz}$ ), are shown in the figure for several values of $\sigma$ and $N_{\mathrm{Db}}$. As expected, the higher the value of $\sigma$, the higher the induced channel depletion and the lower the current level, Fig. 2(a). The frequency and amplitude of the oscillations are also decreased, and even disappear for the highest $\sigma$ due to the excessive channel depletion.

Concerning the influence of $N_{\mathrm{Db}}$, when $\sigma$ is kept constant, the current is higher as $N_{\mathrm{Db}}$ increases because of the higher electron concentration, Fig. 2(b). Additionally, the inset shows that the Gunn oscillations are reinforced by a higher $N_{\mathrm{Db}}$ due to a stronger feedback provided by the electric field allowing the onset and development of such current oscillations. It is interesting to note that this also makes the oscillation frequency increase.

The influence of the channel length and the operation temperature is presented in Fig. 3. As observed, the oscillation frequency essentially scales with the channel length $L(150 \mathrm{GHz}$ for $2 \mu \mathrm{m})$, while the $I-V$ curve is less affected. Just a change in the slope for low voltages is observed while the same saturation current is reached for high forward voltages. We can confirm that, remarkably, Gunn oscillations still remain even with a strong heating of the diodes (uniform temperature of $500 \mathrm{~K}$ considered in the simulations), conditions expected to occur in these small devices in normal operation (under very high voltages and large dissipated power). At $500 \mathrm{~K}$ only a drop of the electron velocity takes place so that the current level lowers and the oscillation frequency decreases (from 300 to $260 \mathrm{GHz}$ ).

In order to analyze the performance of SSDs operating as emitters, the DC to AC conversion efficiency, $\eta$, has been evaluated by means of the superposition of a single-tone 
sinusoidal potential of amplitude $V_{\mathrm{AC}}$ to a DC bias $V_{\mathrm{DC}}$. The dependence of $\eta$ on the frequency of the AC excitation when varying several parameters is shown in Figs. 4 and 5. We have firstly considered four different values for $V_{\mathrm{AC}}(1,5,10$ and $20 \mathrm{~V})$ keeping constant $V_{\mathrm{DC}}=50 \mathrm{~V}$ [Fig. 4(a)], and then used a variable $V_{\mathrm{DC}}(40,50$ and $60 \mathrm{~V})$ while $V_{\mathrm{AC}}$ is set at $10 \mathrm{~V}$ [Fig. 4(b)]. Several bands of AC generation, with a typical maximum efficiency between 0.1 and $0.5 \%$, are observed at the fundamental frequency of the Gunn effect and also at around double frequency. Even if these values are not very high, as GaN diodes are able to support a large DC power, the AC power they can deliver could still be of practical use at such high frequencies.

As expected, the efficiency depends on the amplitude of both the DC and AC excitation. In particular, lower efficiency at generation bands of higher frequencies is observed for high amplitudes of AC excitation [see Fig. 4(a)], while the opposite behavior is observed for the DC amplitude, i.e. higher efficiency is found for higher frequency bands as $V_{\mathrm{DC}}$ increases [see Fig. 4(b)].

The influence of $N_{\mathrm{Db}}, L, T$ and $\sigma$ on the efficiency is shown in Figs. 4(c)-4(d). Similarly to the previous results when just a DC excitation is applied, higher values of $N_{\mathrm{Db}}$ reinforce the oscillations, thus improving the efficiency. When increasing the temperature of operation a shift of the emission bands to lower frequencies takes place because of the smaller drift velocity of electrons at higher temperature, but the efficiency is not degraded. When comparing the efficiency for diodes with different channel lengths, wider emission bands are observed for shorter channels, with the frequency scaling as $1 / L$. This result opens the possibility to increase the emission frequency of SSDs by reducing the channel length. Finally, a higher surface charge

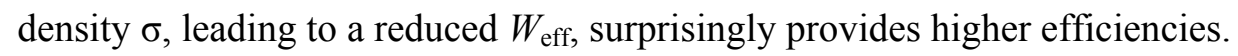

In all the cases, intriguingly, the frequency of the oscillations observed when the SSDs are biased with just a DC voltage (around $300 \mathrm{GHz}$ for $L=1 \mu \mathrm{m}$ ) lies within the second generation band and not the fundamental one (around $150 \mathrm{GHz}$ ) of the oscillations taking place in the presence of an additional AC bias.

In order to explain the previous results and to illustrate the microscopic processes leading to the presence of generation at different bands, we will study a diode where four emission bands are clearly identified, as shown in Fig. 5. Figure 6 shows the profiles of carrier concentration and electric field along the centre of the channel: i) at one given time for different frequencies of the AC excitation (at the centre of each generation band), Figs. 6(a)-6(b), and ii) at different equidistant time moments during one period of excitation with an AC signal of $310 \mathrm{GHz}$ (central 
frequency of the fourth generation band), Figs. 6(c)-6(d). As observed, an increasing number (from 1 to 4) of charge domains (high field regions) drift along the channel for increasing frequencies of the $\mathrm{AC}$ excitation at the centre of each generation band. These domains are originated by the transfer of electrons from the $\Gamma_{1}$ to $U$ valleys (with higher effective mass) in the conduction band of GaN, as occurs in standard GaAs Gunn diodes. It is also noteworthy that the peak frequencies of the higher generation bands are not exactly integer multiples of the fundamental oscillation because the formation of more than one accumulation domain increases the "dead space" (distance needed for the formation of the domain) at the entrance of the SSD.

The remarkably high values of the electric field observed in Fig. 6 do not pose any problem for the correct simulation of the carrier dynamics even if the MC model does not include impact ionization processes, since the electron energy is not still high enough for generating a significant number of electron-hole pairs. Indeed, such electric fields are still well below the breakdown field of Wurtzite GaN.

\section{COMPARISON WITH EXPERIMENTAL RESULTS: MC SELF-CONSISTENT SURFACE CHARGE MODEL}

In order to fix the value of the fitting parameters of the MC model, $\sigma$ and $N_{\mathrm{Db}}$, we have compared our simulations with experimental measurements obtained from the first run of $\mathrm{GaN}$ SSDs fabricated at IEMN following the guidelines of MC results. The devices are made on an epitaxial layer consisting of $1.8 \mu \mathrm{m}$ of undoped hexagonal GaN ([0001] orientation) on a HR Si substrate, with a barrier of $23 \mathrm{~nm}$ of $\mathrm{Al}_{0.3} \mathrm{Ga}_{0.7} \mathrm{~N}$ and covered by $5 \mathrm{~nm}$ of SiN for passivation. We have decided to use $\mathrm{GaN}$ on $\mathrm{Si}$ because of its lower price, in spite of the poorer thermal conductivity since, as confirmed by the results shown in the previous section, heating should not be a critical issue for the onset of Gunn oscillations (even if the temperature increase has an important effect in the $I-V$ curves). Measured values of sheet carrier density $n_{s}$ in this epilayer are in the range $6-8 \times 10^{12} \mathrm{~cm}^{-2}$, with a Hall mobility around $1200 \mathrm{~cm}^{2} / \mathrm{Vs}$. The fabrication process starts by depositing ohmic contacts $(\mathrm{Ti} / \mathrm{Al} / \mathrm{Ni} / \mathrm{Au}$, with a resistance of $0.3 \Omega \mathrm{mm})$ and isolation by ionic implantation $\left(\mathrm{He}^{+}\right)$. Then, the etching of the trenches takes place (with a PMMA resist

and ICP chlorine based technology), and finally the top metal layer ( $\mathrm{Ti} / \mathrm{Pt} / \mathrm{Au})$ is deposited for CPW line access. Several sizes and arrays of SSDs have been processed. A strong technological effort has been necessary in order to obtain a deep etch $(45 \mathrm{~nm})$ together with a small recess width (50 to $100 \mathrm{~nm}$ ), as shown in Figs. 7(a)-7(b). Figure 7(a) is a SEM view of an array of 16 SSDs in parallel and Fig. 7(b) a FIB cut view showing the shape of the trenches. We want to 
remark that this is a completely new technological process and these are the first ever SSDs fabricated on GaN. The electrical characterization of several topologies (different number of channels in parallel: 1, 4, 16 and 32 SSDs) are shown in Figs. 8(a)-8(b). The correct linear law of the current versus the number of SSDs in parallel, inset of Fig. 8(a), shows that there is no apparent thermal limitation associated to the Si substrate. MC simulations also indicate that there is not a phase mismatch between the possible Gunn oscillations within the individual channels [22]. Moreover, the dependence of the $I-V$ curves on the length and width of the channels, Fig. 8(b), follows the trends observed in simulations, what validates the quality of the fabrication process. From these results we have estimated the width of the depletion region $W_{d}$, obtaining a value of $25 \pm 5 \mathrm{~nm}$ for both $1 \mu \mathrm{m}$ and $2 \mu \mathrm{m}$ channels. Then, taking into account that $W_{d}=\sigma / N_{\mathrm{Db}}$ within the constant surface charge model, we have performed simulations using several values of $\sigma$ and $N_{\mathrm{Db}}$, always providing a ratio of $25 \mathrm{~nm}$. The obtained comparison with the experimental results is shown in Fig. 9. As observed, for the lowest values of $\sigma$ and $N_{\mathrm{Db}}$ it is possible to fit the ohmic conductance (slope at low voltage), and for the highest values it is possible to obtain a similar saturation current (both for forward and reverse bias). However, there is no set of parameters able to correctly reproduce the whole $I-V$ curve (even without keeping the ratio of $25 \mathrm{~nm}$, results not shown), thus indicating that the simple constant surface charge model used in these MC simulations is not adequate for the modeling of these devices.

The fact that the constant surface charge model used so far in the simulations is unable to reproduce the experimental results indicates that the surface charge density along the boundary of the trenches is strongly non-homogeneous and voltage dependent, thus leading to the low saturation voltages and currents measured in the SSDs. Other possible reason for the discrepancies could be heating effects, not self-consistently considered in the MC simulations. As pointed out in Sec. II, to account for the dependence of the surface charge on the position and applied voltage, we have to make use of the self-consistent model. Basically, this model allows a local auto-adaptation of the surface charge at the boundaries according to the evolution of the carrier concentration in the conductive channel, which depends on position and bias. As a consequence the effective channel width is locally modulated, and in a different way for each applied voltage. Figure 10 compares the MC results obtained for SSDs with $L=1$ and $2 \mu \mathrm{m}$ and $W=75$ and $150 \mathrm{~nm}$ with the measured $I-V$ curves in devices with the same geometry $\left(N_{\mathrm{Db}}=1 \times 10^{17} \mathrm{~cm}^{-3}\right.$ is considered). MC simulations are now able to satisfactorily reproduce the experimental behavior of the current, both for high-bias (saturation) and low-bias, and for SSDs with different values of channel length and width. The value used to convert the current densities 
(in $\mathrm{A} / \mathrm{m}$ ) obtained from the $2 \mathrm{D}$ simulator to absolute currents (in $\mathrm{A}$ ) is the transversal size that provides the experimental sheet carrier density of the layer structure $\left(n_{s}\right)$, thus obtained as $Z=n_{s} / N_{\mathrm{Db}}$. In this particular case we are using $n_{s}=8 \times 10^{12} \mathrm{~cm}^{-2}$, well within the range of experimental values measured in our epilayers. Even if the low-voltage conductance and the saturation current are not perfectly reproduced, the comparison is much more favorable than with the constant surface charge model. A more satisfactory fitting could be obtained considering the exact dimensions (underetch of about $20 \mathrm{~nm}$ has been detected in the SEM images of the devices) instead of the nominal ones used in the CAD tool. Heating effects (not considered here) could also be important, mainly for the higher current levels.

In order to understand the effect of the self-consistent charge model, in Fig. 11 the profiles of the surface charge (or, equivalently, theoretical induced depletion $W_{d}$ ) for different bias and geometries are shown. It can be observed that they are non-homogeneous and strongly dependent on the applied voltage. The self-adaptation of the surface charge provides values of $W_{d}$ about 25-30 $\mathrm{nm}$ at equilibrium, Fig. 11(a), very close to those obtained from the experimental measurements, thus supporting the physical consistency of this model. The evolution of the profiles of $W_{d}$ with the applied voltage, reported in Fig. 11(b), explains the saturation of the current for voltages lower than in the case of using the constant surface charge model, and the lower value reached by the current. Since the voltage applied to the anode contact $V_{\text {bias }}$ reaches the sidewalls of the trenches surrounding the channel, it acts similarly to the gate voltage in a field effect transistor. This means that by increasing $V_{\text {bias }}$ the carrier concentration in the channel should increase. That is what happens with the constant surface charge model, in which the current increases with positive $V_{\text {bias }}$ and only for very high voltages a trend to saturation is observed due to electron velocity saturation effects in GaN (see, e.g., Fig. 2). However, in the case of the self-consistent model, when the carrier concentration tends to increase because of a higher $V_{\text {bias, }}$, the surface charge also increases, as observed in Fig. 11(b), due to the presence of more carriers close to the boundaries of the trenches. The final result is that the influence of an increase of $V_{\text {bias }}$ on the carrier concentration in the channel is compensated by an enhanced $\sigma$, so that a saturation regime is reached in the current, taking place for values of $V_{\text {bias }}$ much lower than with constant surface charge. The increase of $\sigma$ with $V_{\text {bias }}$ is also non-homogeneous along the channel, being more pronounced at the entrance for low voltages, while for voltages beyond the threshold for intervalley transfer is more significant at the anode side of the channel, where intervalley mechanisms lead to an accumulation of electrons. As a consequence of the presence of an enhanced surface charge, the channel region close to the trenches is depleted even for high 
voltages applied to the anode, thus localizing the current flux along the centre. This is opposite to what is found in the simulations using the constant surface charge model (not shown), in which once the applied voltage has compensated the depletion originated by the constant $\sigma$, more and more carriers are attracted towards the sidewalls by the positive $V_{\text {bias}}$, and electrons accumulate in their vicinity.

The role played by the charges present at the etched surfaces was known to be essential in the behavior of the SSDs [15], but these results show an unexpectedly strong influence, mainly due to the high applied voltages (much higher than in previously fabricated GaAs- or InGaAsbased SSDs). Indeed, the geometry of the first fabrication batch of diodes was designed with narrow channels $(75$ and $150 \mathrm{~nm})$ expecting to have an electron concentration in the channel high enough for the onset of oscillations (relying on the results obtained with the constant surface charge model shown in the previous section). However, unfortunately both experimental measurements (S parameters up to $325 \mathrm{GHz}$ ) and $\mathrm{MC}$ simulations with the self-consistent model exhibited no oscillations due to an insufficient electron density in the channel. Thus, the width of the channels must be increased in order to achieve the onset of Gunn oscillations. As an example, Fig. 12 shows results for diodes with larger widths (up to $750 \mathrm{~nm}$ ), always keeping a channel length of $1 \mu \mathrm{m}$, obtained with the MC simulator using the self-consistent surface charge model. It is observed that current oscillations only appear for channels wider than $500 \mathrm{~nm}$, with threshold voltages of about 40-50 V and frequencies around $300 \mathrm{GHz}$. In these diodes with wider channels, MC results including the self-consistent charge model exhibit the same qualitative dependencies and basic operation as those discussed in Sec. III, obtained with constant charge. Other approaches that may allow the onset of Gunn oscillations by modifying the geometry of the SSDs are being investigated at this moment and, together with wider channels, will be considered in next fabrication batches.

In [31], low-frequency noise measurements were suggested as an indicator of highfrequency oscillations as illustrated by calculations performed, with the constant surface charge model, in a reference GaN SSD with a geometry ( $1 \mu \mathrm{m}$ long, $80 \mathrm{~nm}$ wide channel) similar to those analyzed here. As explained above, because of surface charge issues, such structures, when fabricated with our technology, have been found unable to generate Gunn oscillations. However, the proposed indicator is expected to be of validity in appropriately designed diodes. 


\section{CONCLUSIONS}

The potentiality of $\mathrm{GaN}$ based planar asymmetric nanodiodes for the generation of $\mathrm{THz}$ signals exploiting Gunn effect has been analyzed. MC simulations have been used to confirm the principle of operation of the diodes and the dependence of their performance on several geometrical and physical parameters. The $1 \mu \mathrm{m}$ channel length SSDs exhibit oscillations for voltages above $30 \mathrm{~V}$ at frequencies higher than $300 \mathrm{GHz}$.

A first batch of diodes (a challenge in GaN technology because of their small dimensions) has been successfully fabricated. However, no oscillation was found due to the strong and unexpected influence of surface charge, that was identified by means of MC simulations (in which a self-consistent charge model was necessary to reproduce the experimental $I-V$ curves). Such a model evidenced the presence of an enhanced surface charge under far-from-equilibrium conditions due to the very high voltages applied to the diodes (necessary for the onset of the oscillations). The depletion induced by the surface charge reduces the carrier concentration in the channel to a level that inhibits the appearance of oscillations.

MC simulations indicate that wider channels where carrier concentration is larger and improved geometries to counteract the influence of surface charge must be used in future fabrication runs in order to achieve the oscillations.

\section{ACKNOWLEDGEMENTS}

This work has been partially supported by the European Commission through the ROOTHz Project ICT-2009-243845, by the Dirección General de Investigación (MICINN) through Project TEC2010-15413. 


\section{REFERENCES}

[1] C. Mann, Proceedings of the IEEE/MTT-S International Microwave Symposium, 1705 (2007).

[2] T. H. Crowe, W. L. Bishop, D. W. Porterfield, J. L. Hesler, and R. M. Weikle, IEEE J. Solid-State Circuits 40, 2104 (2005).

[3] J. B. Gunn, Solid State Commun., 1, 88 (1963).

[4] E. Alekseev and D. Pavlidis, Solid-State Electronics 44, 941-947 (2000).

[5] K. Mutambaa, O. Yilmazoglua, C. Sydloa, M. Mira, S. Hubbardb, G. Zhaob, I. Daumillerc and D. Pavlidis, Superlatt. Microstruct., 40, 363, (2006).

[6] O. Yilmazoglu, K. Mutamba, D. Pavlidis and T. Karaduman, Electronics Lett., 43, 480, (2007).

[7] O. Yilmazoglu, K. Mutamba, D. Pavlidis and T. Karaduman, IEEE Trans. Electron Dev., 55, 1563, (2008).

[8] N. J. Pilgrim, A. Khalid, G. M. Dunn, and D. R. S. Cumming, Semicond. Sci. Technol. 23, 075013 (2008).

[9] Ch. Li, A. Khalid, N. J. Pilgrim, M. C. Holland, G. M. Dunn, and D. R. S. Cumming, 35th International Conference on Infrared Millimeter and Terahertz Waves, 2010. DOI: 10.1109/ICIMW.2010.5612507.

[10] A. M. Song, M. Missous, P. Omling, A. R. Peaker, L. Samuelson and W. Seifert, Appl. Phys. Lett. 83, 1881 (2003).

[11] C. Balocco, A. M. Song, M. Aberg, A. Forchel, T. González, J. Mateos, I. Maximov, M. Missous, A. A. Rezazadeh, J. Saijets, L. Samuelson, D. Wallin, K. Williams, L. Worschech and H. Q. Xu, Nano Letters 5, 1423 (2005).

[12] C. Balocco, S. R. Kasjoo, X. F. Lu, L. Q. Zhang, Y. Alimi, S. Winnerl and A. M. Song, Appl. Phys. Lett. 98, 223501 (2011).

[13] C. Balocco, M. Halsall, N. Q. Vinh and A. M. Song, J. Phys.: Condens. Matter 20, 384203 (2008).

[14] C. Jacoboni and P. Lugli, The Monte Carlo Method for Semiconductor Device Simulation, Vienna, Springer, 1989. 
[15] Mateos J., Vasallo B. G., Pardo D. and González T., Appl. Phys. Lett. 86, 212103 (2005).

[16] K. Y. Xu, X. F. Lu, A. M. Song and G. Wang, Appl. Phys. Lett. 92, 163503 (2008).

[17] I. Iñiguez-de-la-Torre, J. Mateos, D. Pardo, A. M. Song and T. González, Appl. Phys. Lett. 94, 093512 (2009).

[18] I. Iñiguez-de-la-Torre, J. Mateos, D. Pardo and González T., J. Appl. Phys. 103, 024502 (2008).

[19] K. Y. Xu, X. F. Lu, A. M. Song and G. Wang, J. Appl. Phys. 103, 113708 (2008).

[20] K. Y. Xu, G. Wang and A. M. Song, Appl. Phys. Lett. 93, 233506 (2008).

[21] T. González, I. Iñiguez-de-la Torre, D. Pardo, J. Mateos and A. M. Song, Journal of Physics: Conference Series 193, 012018 [1-4] (2009).

[22] T. González, I. Íñiguez-de-la-Torre, D. Pardo, A. M. Song and J. Mateos, 22th International Conference on Indium Phosphide and Related Materials (IPRM 2010), Takamatsu, Japan, May 31-June 4, (2011).

[23] T. Sadi, F. Dessene and J-L Thobel, J. Appl. Phys. 105, 053707 (2009).

[24] J. Mateos, B. G. Vasallo, D. Pardo, T. González, J. S. Galloo., Y. Roelens, S. Bollaert and A. Cappy, Nanotechnology 14, 117 (2003).

[25] J. Mateos, B. G. Vasallo, D. Pardo, T. González, J. S. Galloo, S. Bollaert, Y. Roelens and A. Cappy, IEEE Trans. Electron Devices 50, 1897 (2003).

[26] I. Iñiguez-de-la-Torre, J. Mateos, T. González, D. Pardo, J. S. Galloo, S. Bollaert, Y. Roelens and A. Cappy, Semicond. Sci. Technol. 22, 663 (2007).

[27] I. Iñiguez-de-la-Torre, J. Mateos, D. Pardo and T. González, J. Appl. Phys. 103, 024502 (2008).

[28] C. Sevik and C. Bulutay, Semicond. Sci. Technol. 19, S188 (2004).

[29] C. Sevik and C. Bulutay, Appl. Phys. Lett. 85, 3908 (2004).

[30] C. Sevik and C. Bulutay, Semicond. Sci. Technol. 19, S188 (2004)

[31] A. Iñiguez-de-la-Torre, I. Iñiguez-de-la-Torre, J. Mateos and T. González, Appl. Phys. Lett. 99, 062109 (2011). 


\section{LIST OF FIGURES}
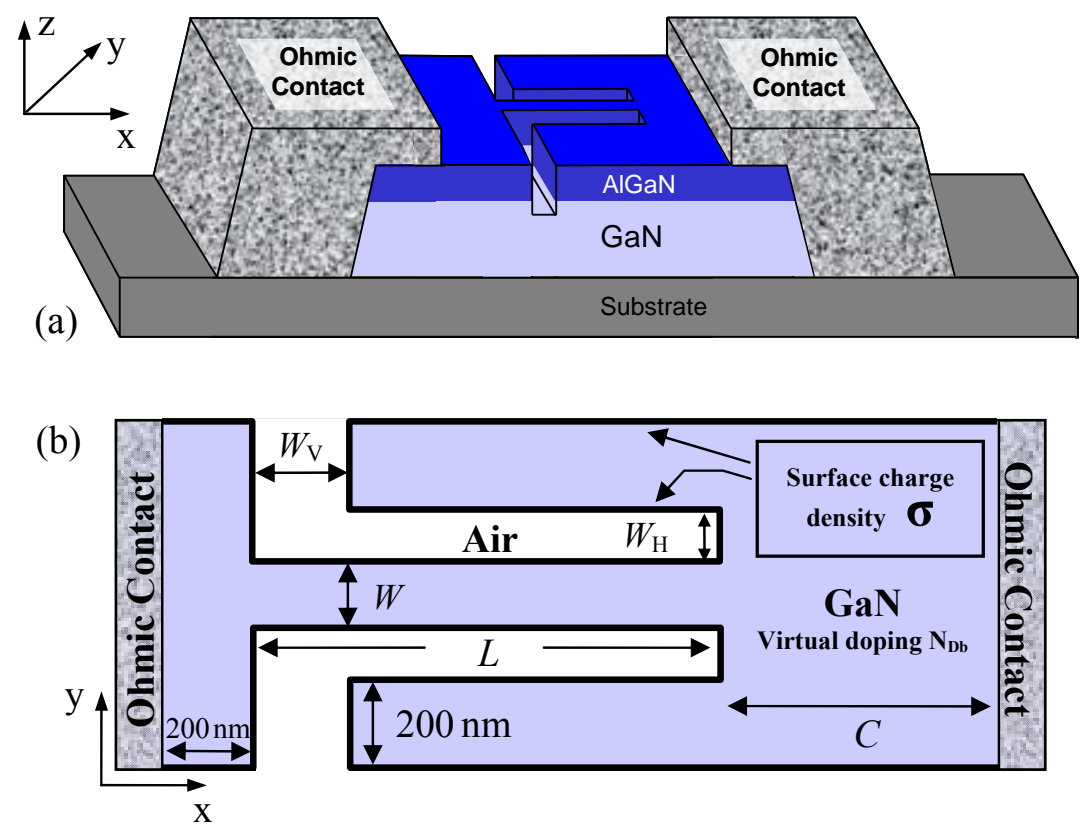

FIGURE 1. (a) Typical geometry of SSDs and (b) scheme of the top-view simulations of the GaN channel with the involved parameters ("virtual" doping $N_{D b}$ and negative surface charge density $\sigma$ ). $L$ and $W$ are the length and width of the channel respectively, $W_{\mathrm{H}}$ the width of the horizontal trenches, $W_{\mathrm{V}}$ the width of the vertical trenches and $C$ the length of the access region. 

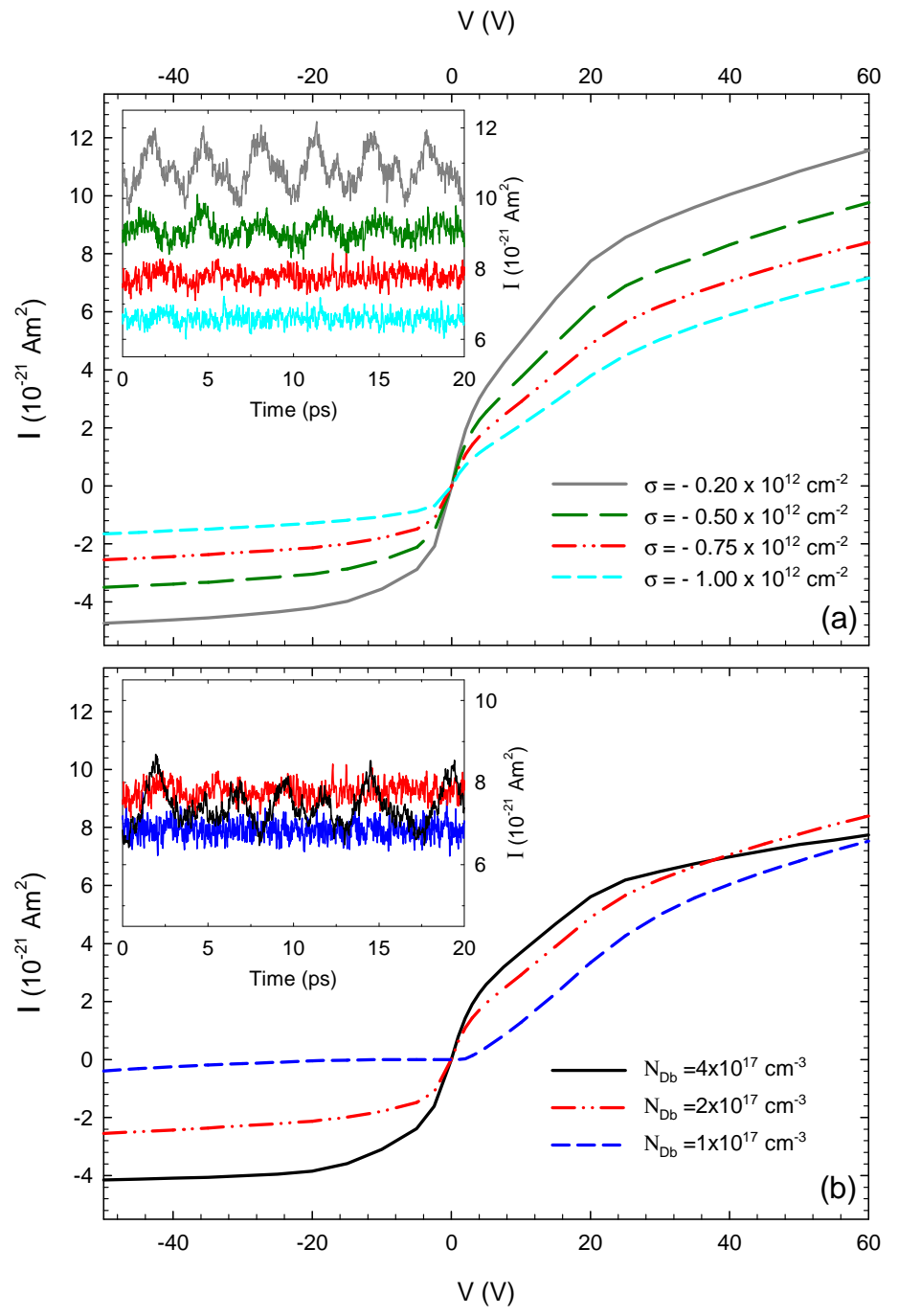

FIGURE 2. $I-V$ curves of the GaN SSDs $\left(L=1 \mu \mathrm{m}, W=150 \mathrm{~nm}, W_{\mathrm{V}}=200 \mathrm{~nm}, W_{\mathrm{H}}=100 \mathrm{~nm}\right.$ and $C=600 \mathrm{~nm}$ ) when varying: (a) the surface charge density $\sigma$ (for $N_{\mathrm{Db}}=2 \times 10^{17} \mathrm{~cm}^{-3}$ ) and (b) the background doping $N_{\mathrm{Db}}$ (for $\sigma=-0.75 \times 10^{12} \mathrm{~cm}^{-2}$ ). The plotted value of the current is obtained as the time average for long simulations, including a large enough number of periods when oscillations are present. The insets show current sequences calculated for an applied voltage of $50 \mathrm{~V}$. 


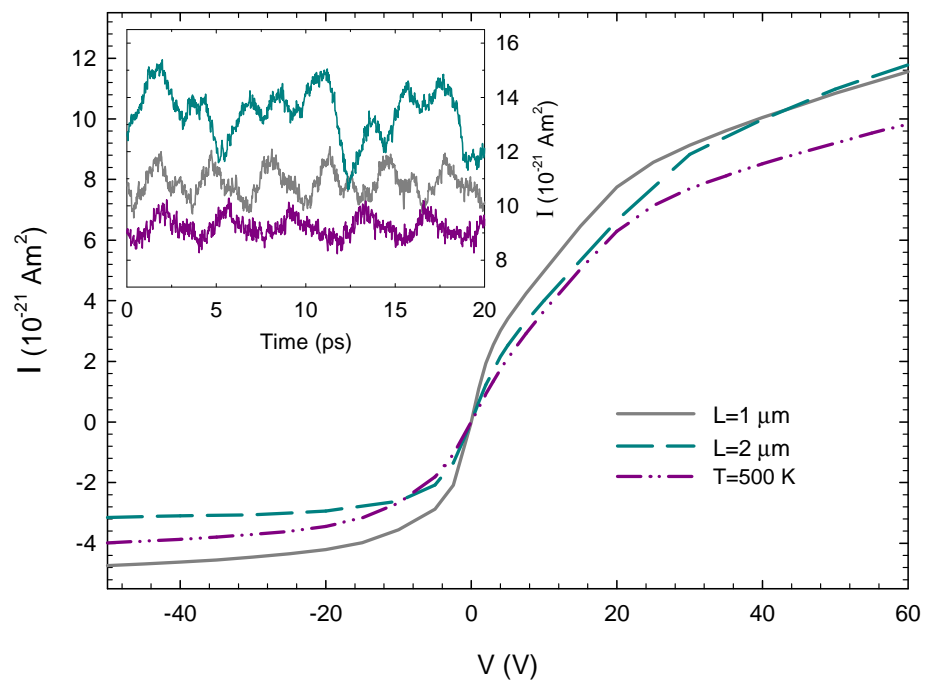

FIGURE 3. $I-V$ curves of the GaN simulated SSDs $\left(\sigma=-0.2 \times 10^{12} \mathrm{~cm}^{-2}, N_{\mathrm{Db}}=2 \times 10^{17} \mathrm{~cm}^{-3}\right.$, $W=150 \mathrm{~nm}, W_{\mathrm{H}}=100 \mathrm{~nm}$ ) when varying the channel length $L$ and the temperature (for $L=1 \mu \mathrm{m}$ ). The inset show current sequences calculated for an applied voltage of $50 \mathrm{~V}(100 \mathrm{~V}$ for $L=2 \mu \mathrm{m})$. 

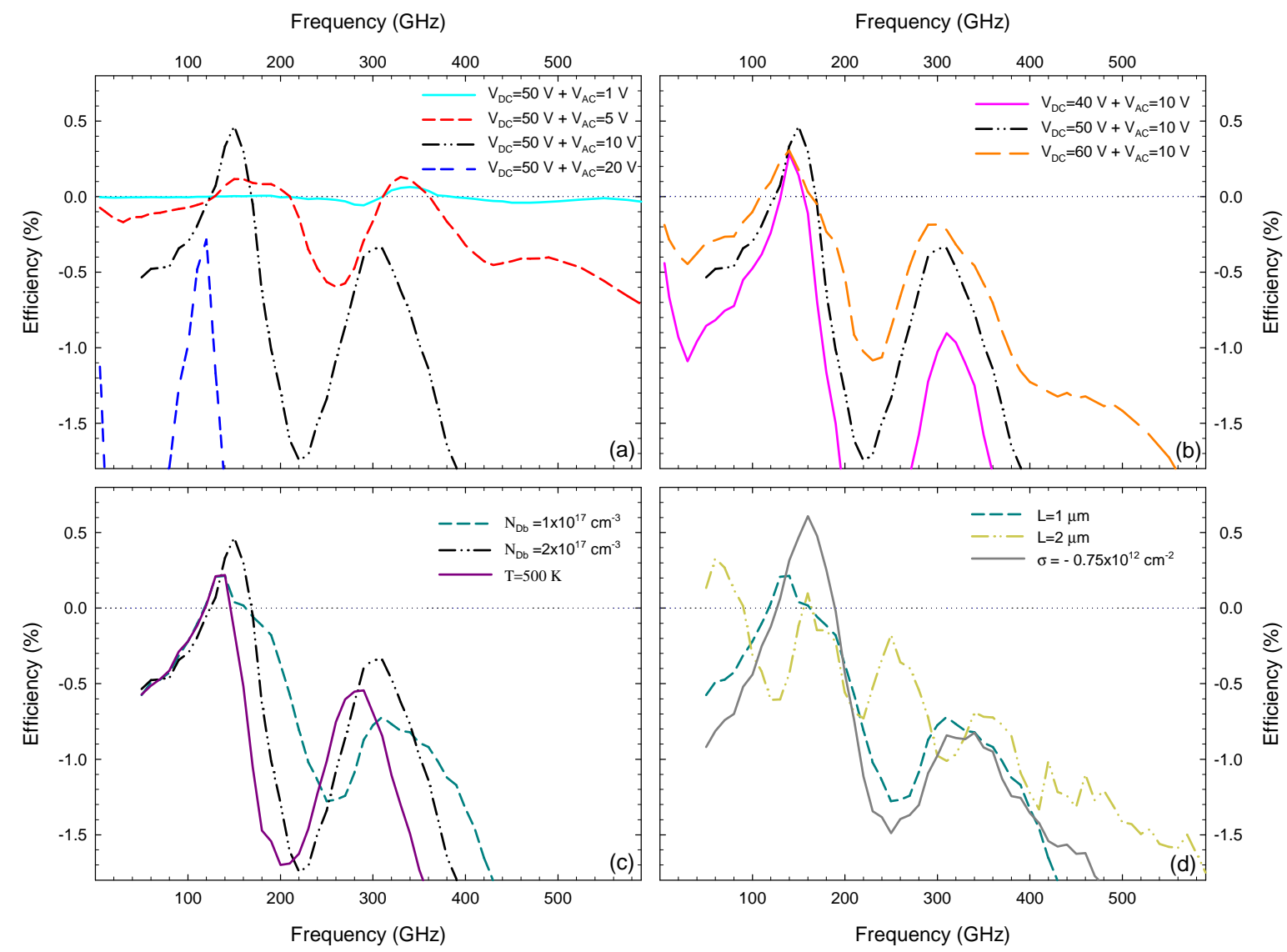

FIGURE 4. DC to AC conversion efficiency $\eta$ as a function of the frequency of the $A C$ excitation when varying: (a) the $\mathrm{AC}$ bias conditions $\left(V_{\mathrm{DC}}=50 \mathrm{~V}\right)$, (b) the $\mathrm{DC}$ bias conditions $\left(V_{\mathrm{AC}}=10 \mathrm{~V}\right)$, (c) background doping $N_{\mathrm{Db}}$ and temperature $T$ and (d) channel length $L$ and surface charge $\sigma\left(N_{\mathrm{Db}}=1 \times 10^{17} \mathrm{~cm}^{-3}\right)$. Unless otherwise indicated, $L=1 \mu \mathrm{m}, W=150 \mathrm{~nm}, W_{\mathrm{V}}=200 \mathrm{~nm}$, $W_{\mathrm{H}}=100 \mathrm{~nm}, C=600 \mathrm{~nm}, \sigma=-0.2 \times 10^{12} \mathrm{~cm}^{-2}, N_{\mathrm{Db}}=2 \times 10^{17} \mathrm{~cm}^{-3}$, and $T=300 \mathrm{~K}$. In (c) and (d) $V_{\mathrm{DC}}=50 \mathrm{~V}$ and $V_{\mathrm{AC}}=10 \mathrm{~V}$ (except for the case $L=2 \mu \mathrm{m}$, for which $V_{\mathrm{DC}}=100 \mathrm{~V}$ and $V_{\mathrm{AC}}=20 \mathrm{~V}$ ). 


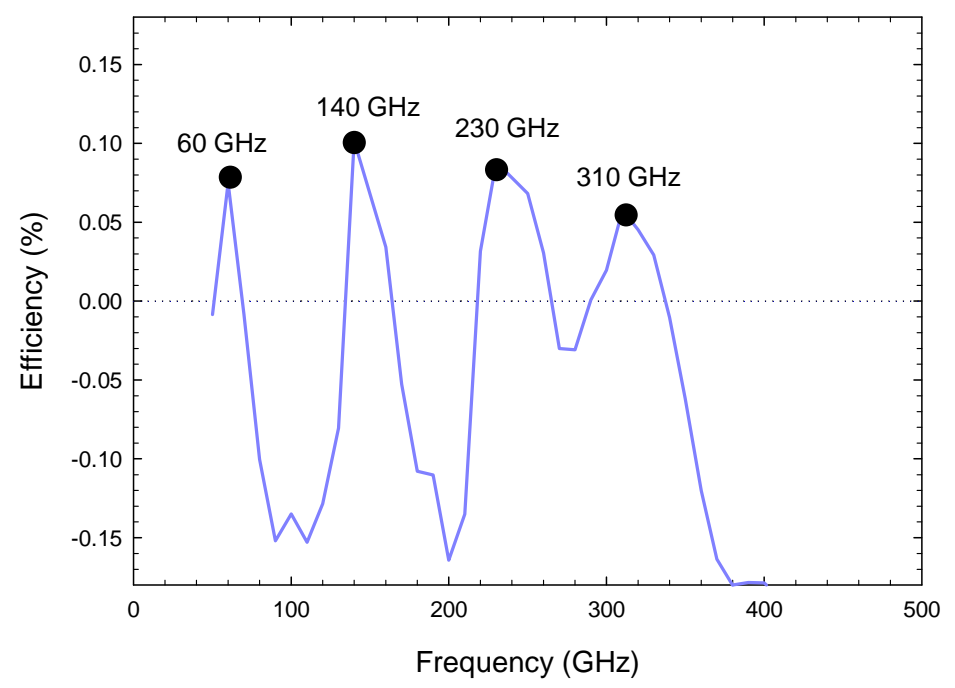

FIGURE 5. DC to AC conversion efficiency $\eta$ as a function of the frequency of the AC excitation for a diode with $L=2 \mu \mathrm{m}, W=150 \mathrm{~nm}, W_{\mathrm{V}}=300 \mathrm{~nm}, W_{\mathrm{H}}=100 \mathrm{~nm}, C=600 \mathrm{~nm}$, $\sigma=-0.2 \times 10^{12} \mathrm{~cm}^{-2}, N_{\mathrm{Db}}=2 \times 10^{17} \mathrm{~cm}^{-3}$, and $T=300 \mathrm{~K} . V_{\mathrm{DC}}=100 \mathrm{~V}$ and $V_{\mathrm{AC}}=7 \mathrm{~V}$. 

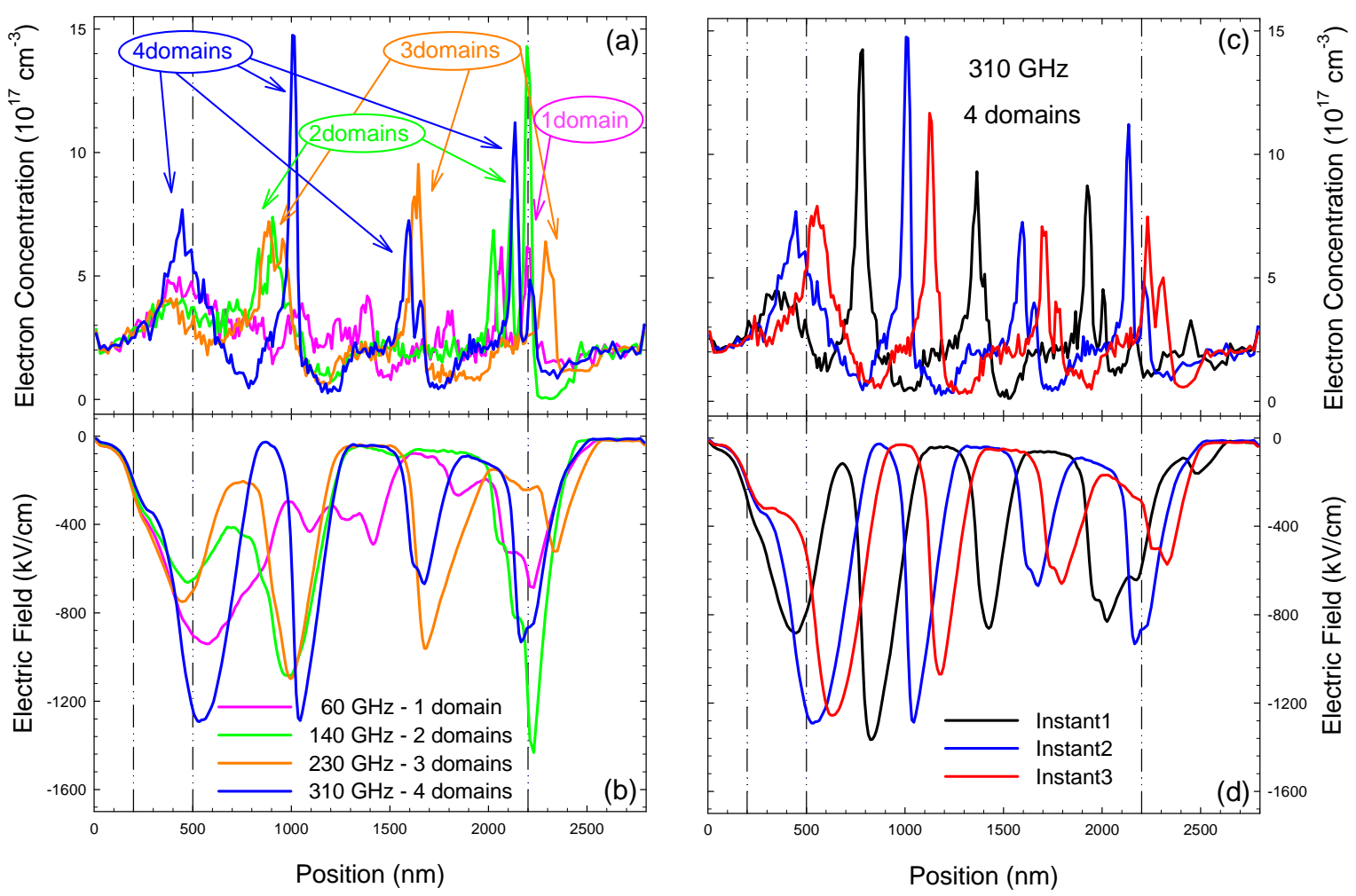

FIGURE. 6. Profiles of (a) and (c) carrier concentration and (b) and (d) electric field along the centre of the channel of one SSD at room temperature for $V_{\mathrm{DC}}=100 \mathrm{~V}$ and $V_{\mathrm{AC}}=7 \mathrm{~V}$. Curves in (a) and (b) correspond to a given time within one period of the AC signal for frequencies at the center of the emission bands observed in Fig. 5 (60, 140, 230 and $310 \mathrm{GHz}$ ). In (c) and (d) they correspond to equidistant time moments during one period of the $\mathrm{AC}$ excitation of frequency $310 \mathrm{GHz}$. The diode has $L=2 \mu \mathrm{m}, W=150 \mathrm{~nm}, W_{\mathrm{V}}=300 \mathrm{~nm}, W_{\mathrm{H}}=100 \mathrm{~nm}, C=600 \mathrm{~nm}$, $\sigma=-0.2 \times 10^{12} \mathrm{~cm}^{-2}$ and $N_{\mathrm{Db}}=2 \times 10^{17} \mathrm{~cm}^{-3}$. Vertical lines indicate the position of the vertical trenches and the end of the channel. 

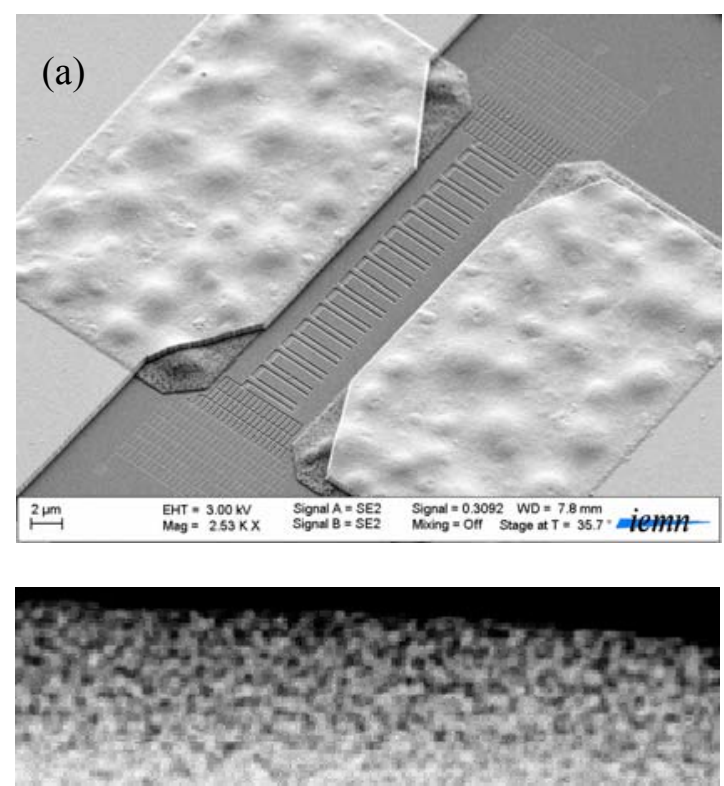

(b)

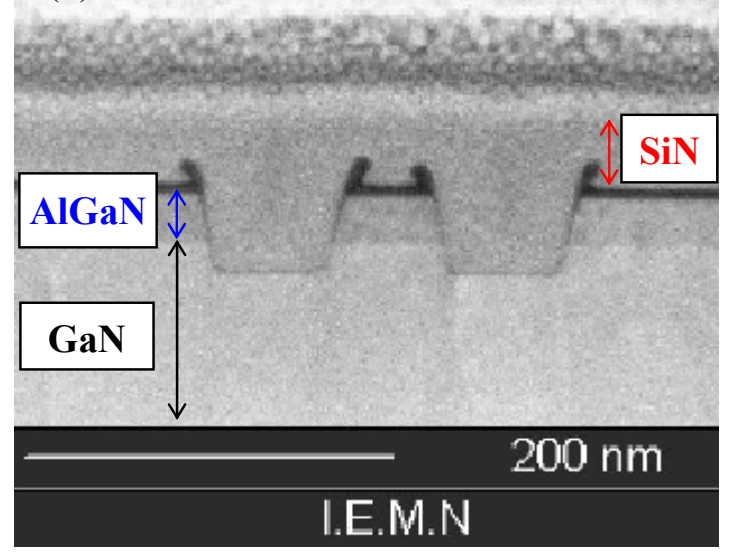

FIGURE 7. (a) SEM image of an array of 16 SSDs in parallel and (b) FIB cut view showing the shape of the recess. 


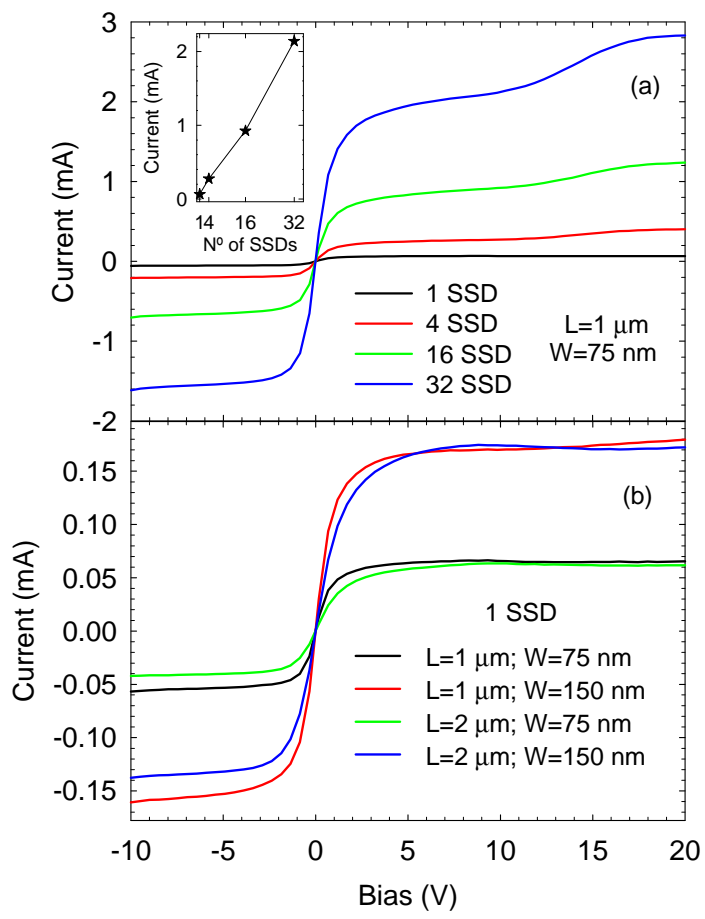

FIGURE 8. Experimental $I-V$ characteristics of (a) arrays of SSDs with different number of diodes in parallel with $L=1 \mu \mathrm{m}, W=75 \mathrm{~nm}$, and (b) of a single SSD with different lengths and widths (all with $W_{\mathrm{V}}=100 \mathrm{~nm}, W_{\mathrm{H}}=50 \mathrm{~nm}, C=400 \mathrm{~nm}$ ). The inset in (a) shows the current for an applied bias of $10 \mathrm{~V}$ as a function of the number of SSDs in parallel. 


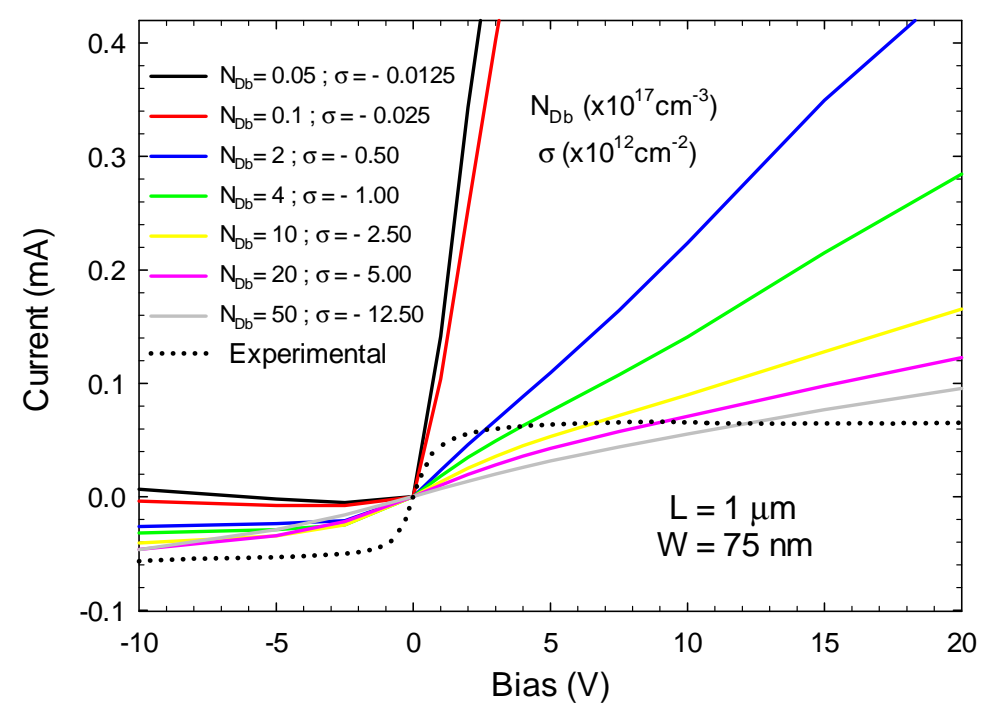

FIGURE 9. Comparison between measured and simulated $I-V$ curves for a diode with $L=1 \mu \mathrm{m}$ and $W=75 \mathrm{~nm}$ with different sets of values of $N_{\mathrm{Db}}$ and $\sigma$ providing the same $W_{\mathrm{d}}=\sigma / N_{\mathrm{Db}}=25 \mathrm{~nm}$. 

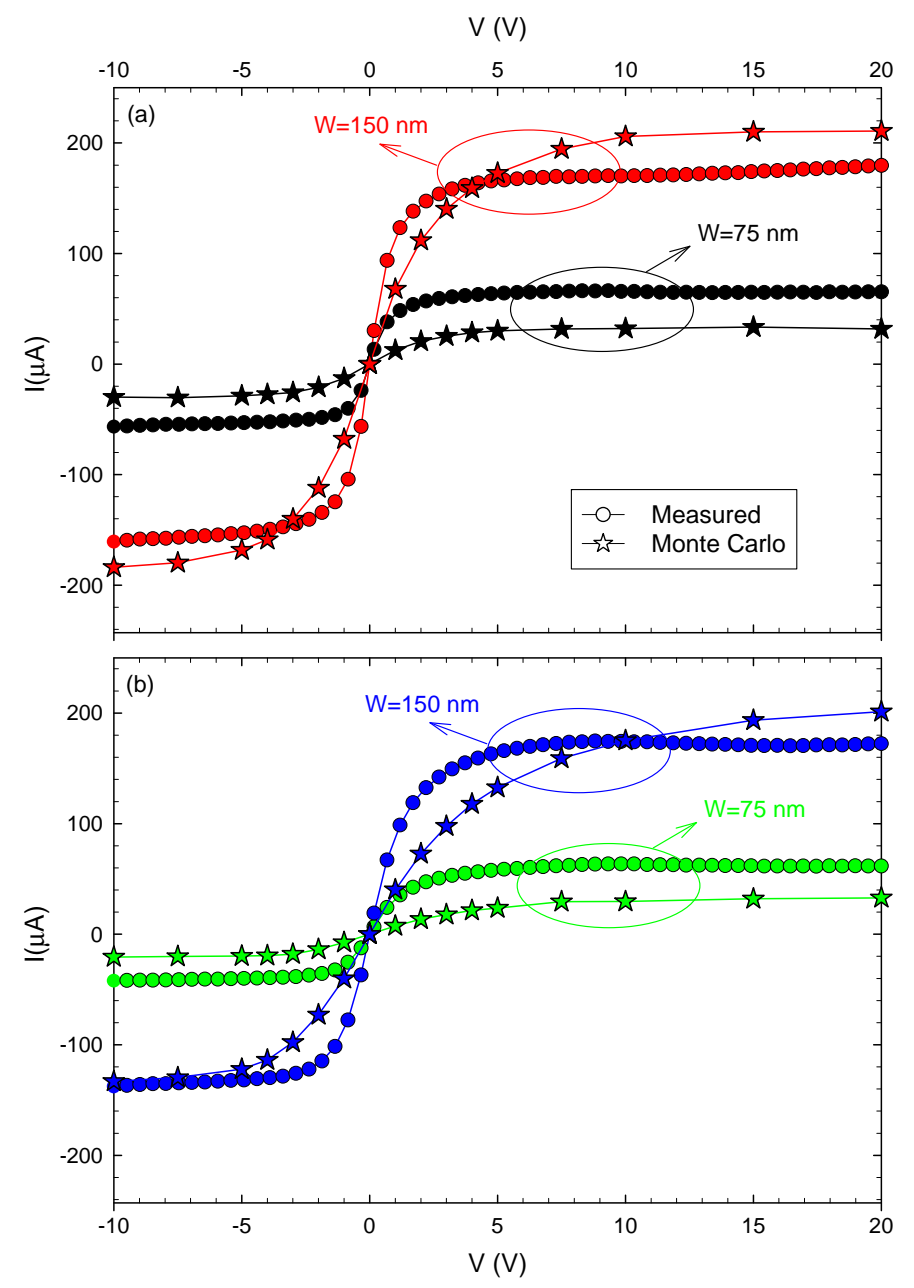

FIGURE 10. Comparison between experimental and simulated $I-V$ curves of SSDs with channel length (a) $1 \mu \mathrm{m}$ and (b) $2 \mu \mathrm{m}\left(W_{\mathrm{V}}=100 \mathrm{~nm}, W_{\mathrm{H}}=50 \mathrm{~nm}, C=400 \mathrm{~nm}\right)$. The self-consistent charge model is used with $n_{s}=8 \times 10^{12} \mathrm{~cm}^{-2}$ and $N_{\mathrm{Db}}=1 \times 10^{17} \mathrm{~cm}^{-3}$. 


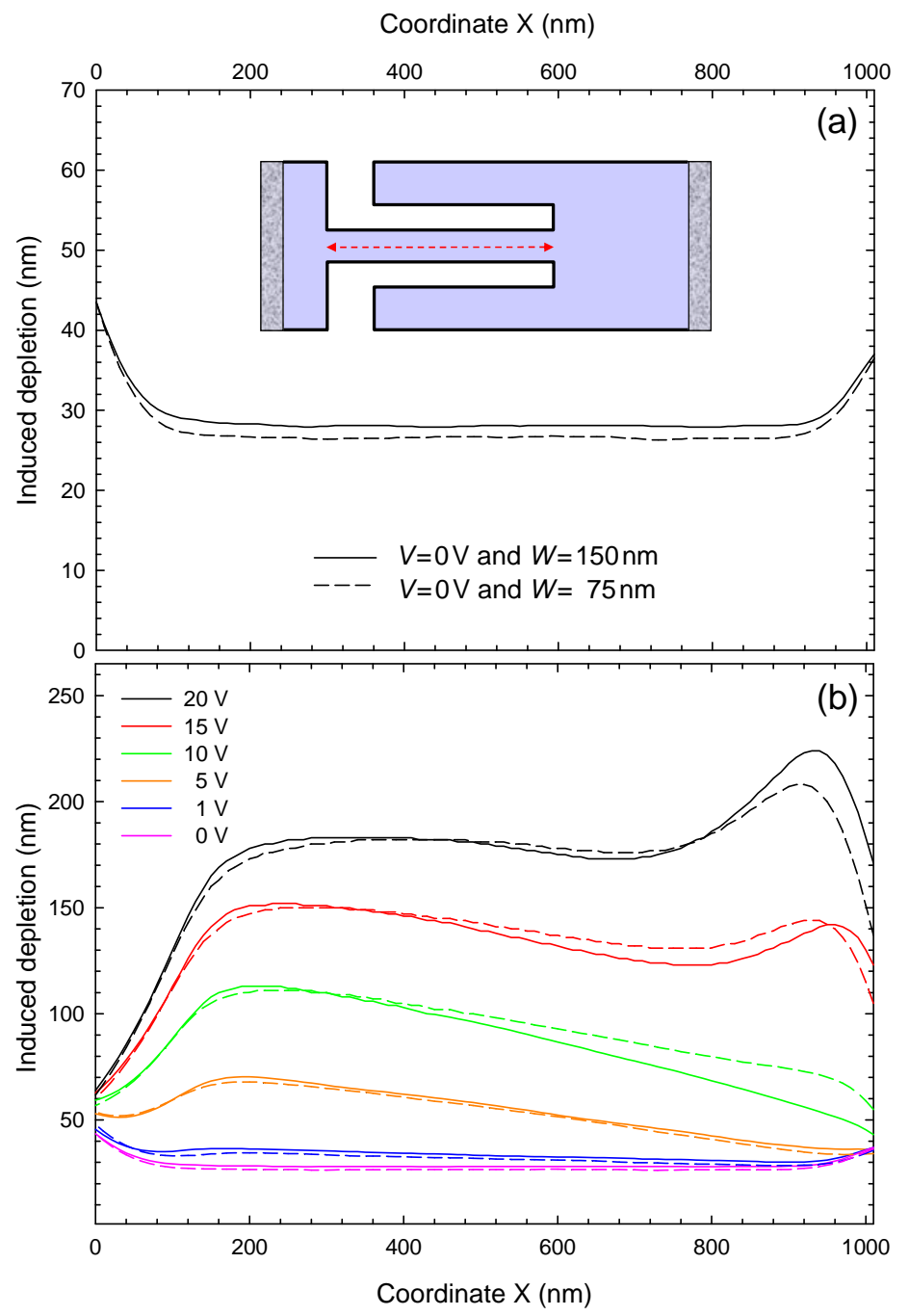

FIGURE 11. Profiles of theoretical induced depletion $W_{d}=\sigma / N_{\mathrm{Db}}$ originated by the surface charge in the diodes of $1 \mu \mathrm{m}$ length and two different widths ( $W=75$ and $150 \mathrm{~nm}$ ). (a) Equilibrium conditions. (b) Positive applied voltages. Solid lines correspond to $W=150 \mathrm{~nm}$ and dashed lines to $\mathrm{W}=75 \mathrm{~nm}$. 


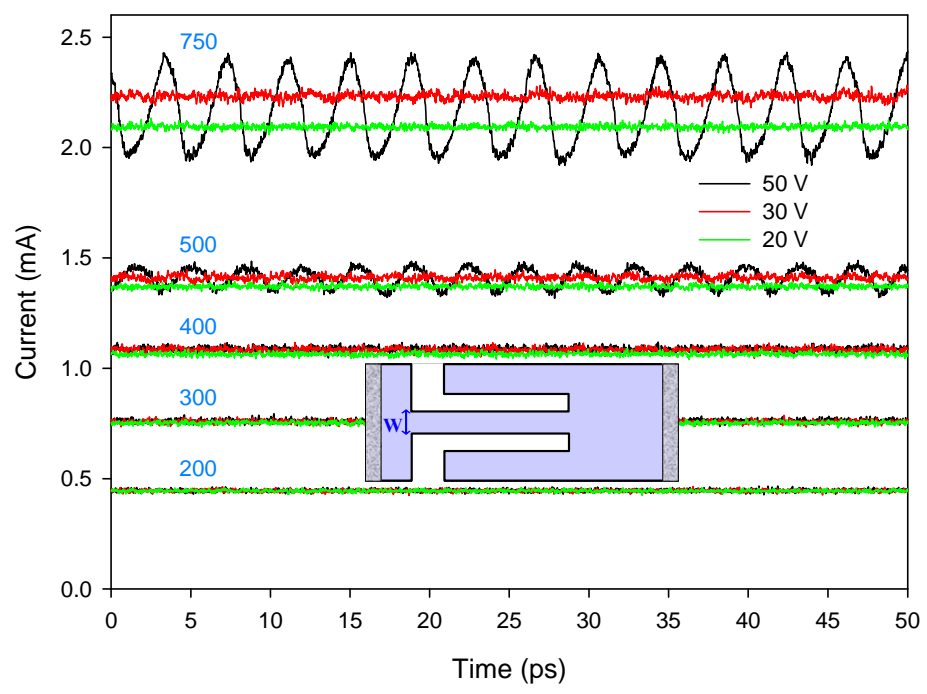

FIGURE 12. Current sequences for diodes with different widths and applied voltages of 20, 30 and $50 \mathrm{~V}$. The channel length is $1 \mu \mathrm{m}$ and the temperature of operation is $300 \mathrm{~K}$. The numbers in the graph correspond to $W$ in $\mathrm{nm}$. The chosen value for $n_{s}$ is $8 \times 10^{12} \mathrm{~cm}^{-2}$. 

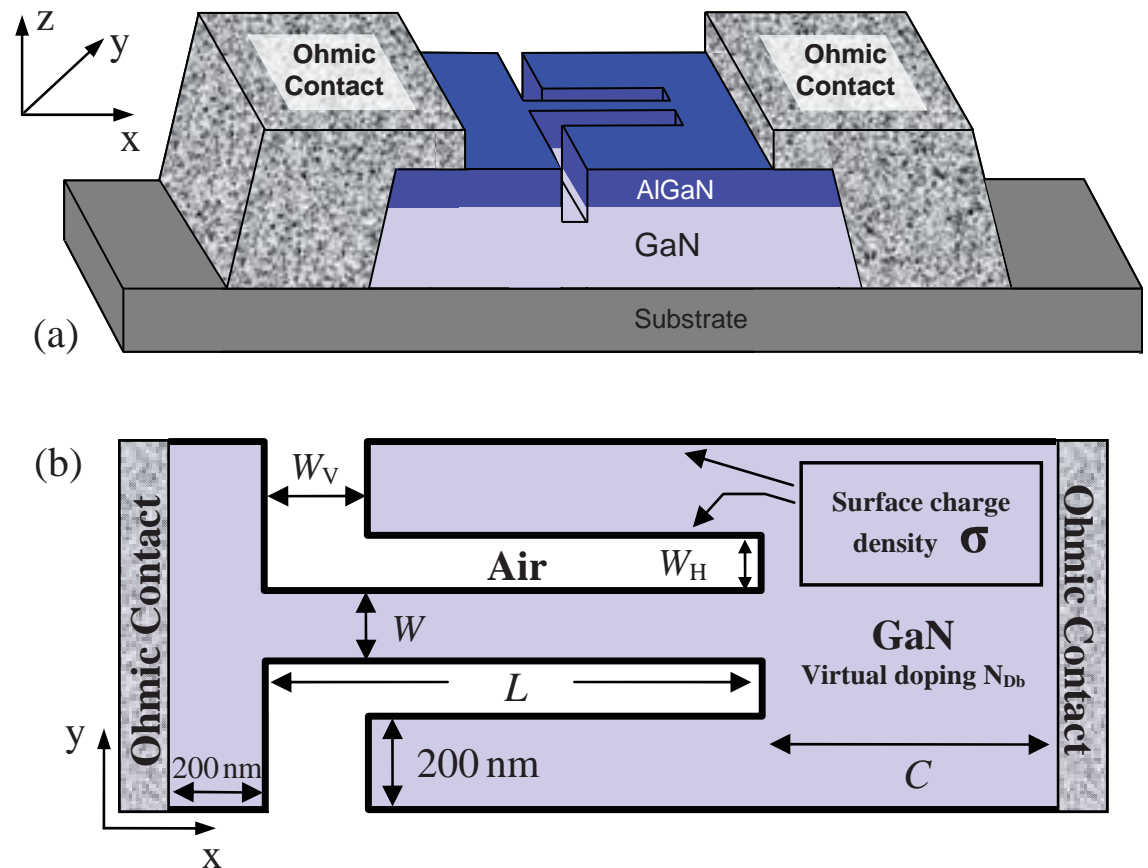

FIG. 1. 


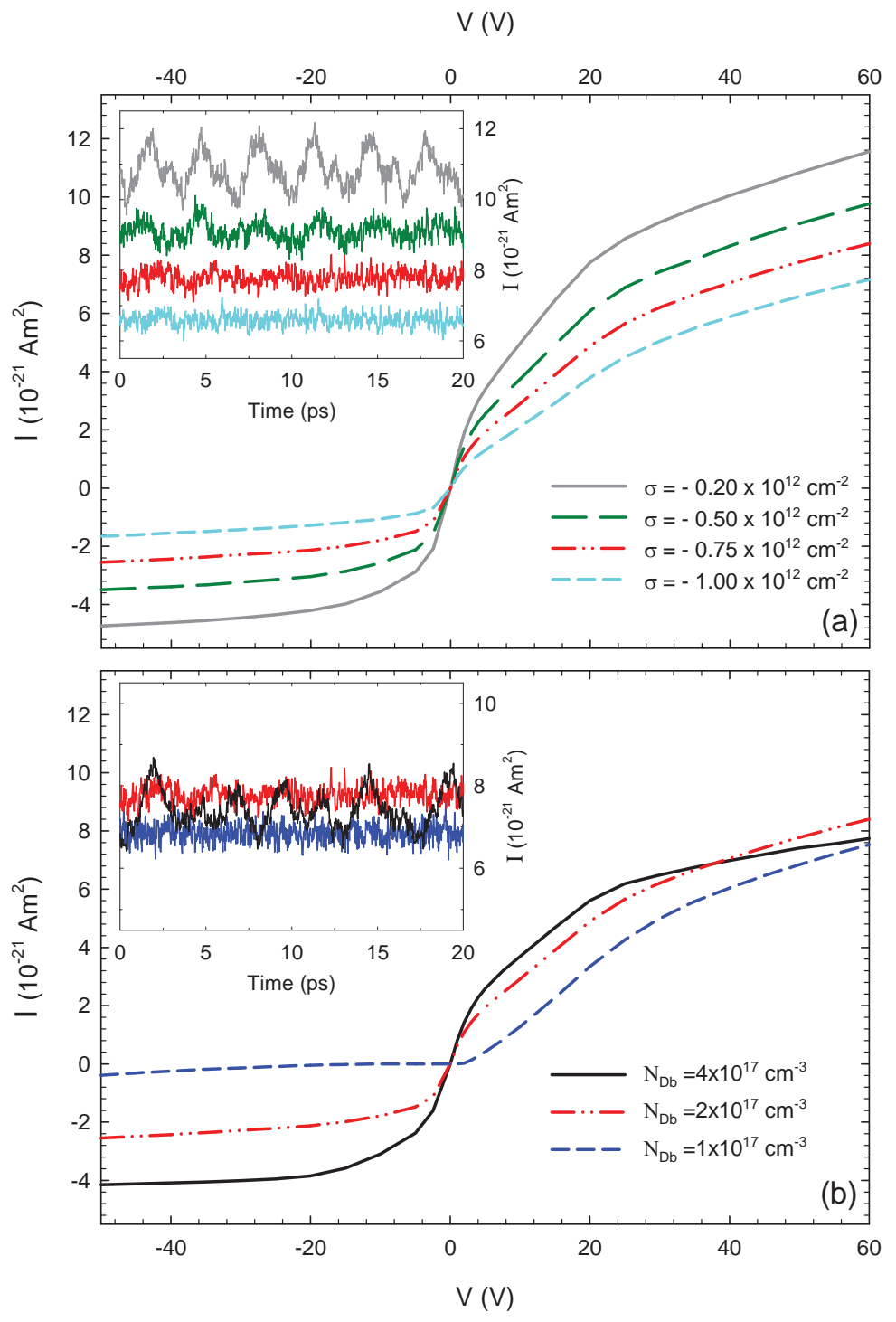

FIG. 2. 


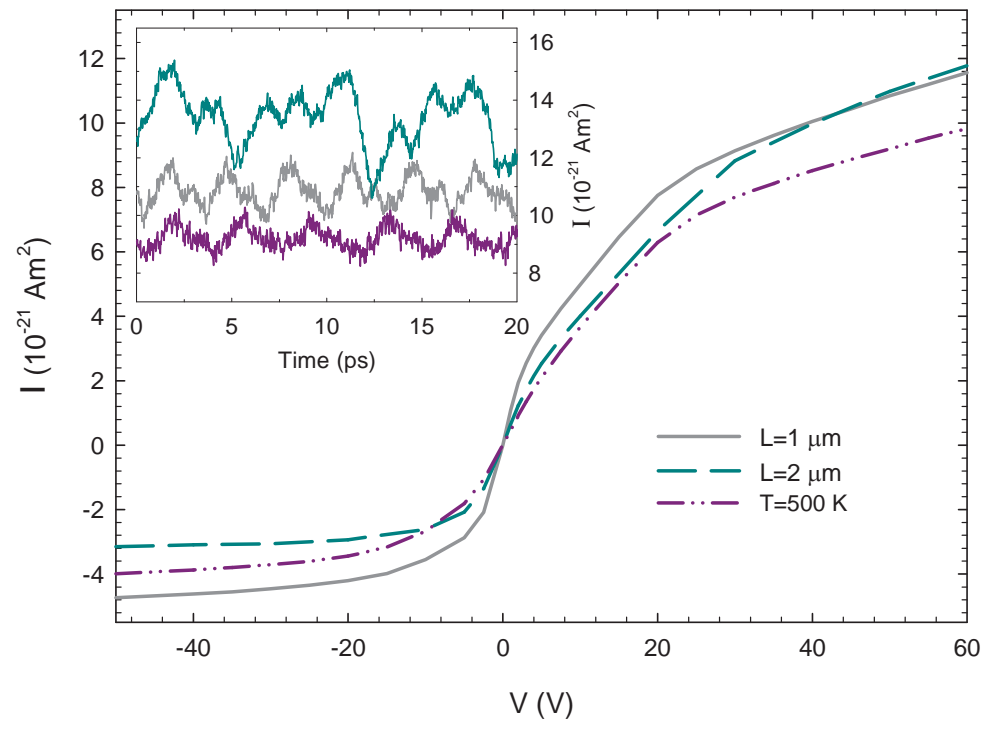

FIG. 3. 


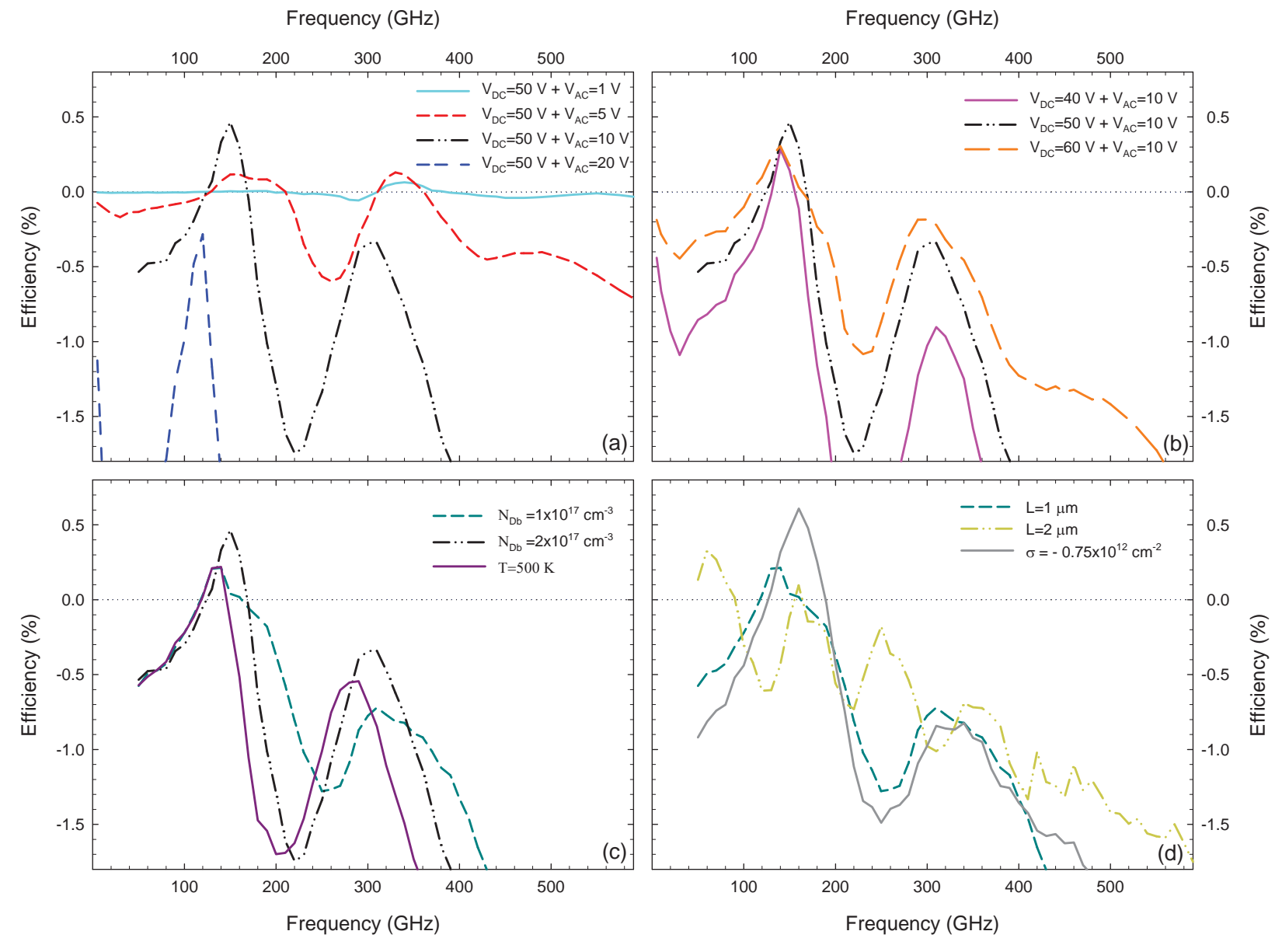

FIG. 4. 


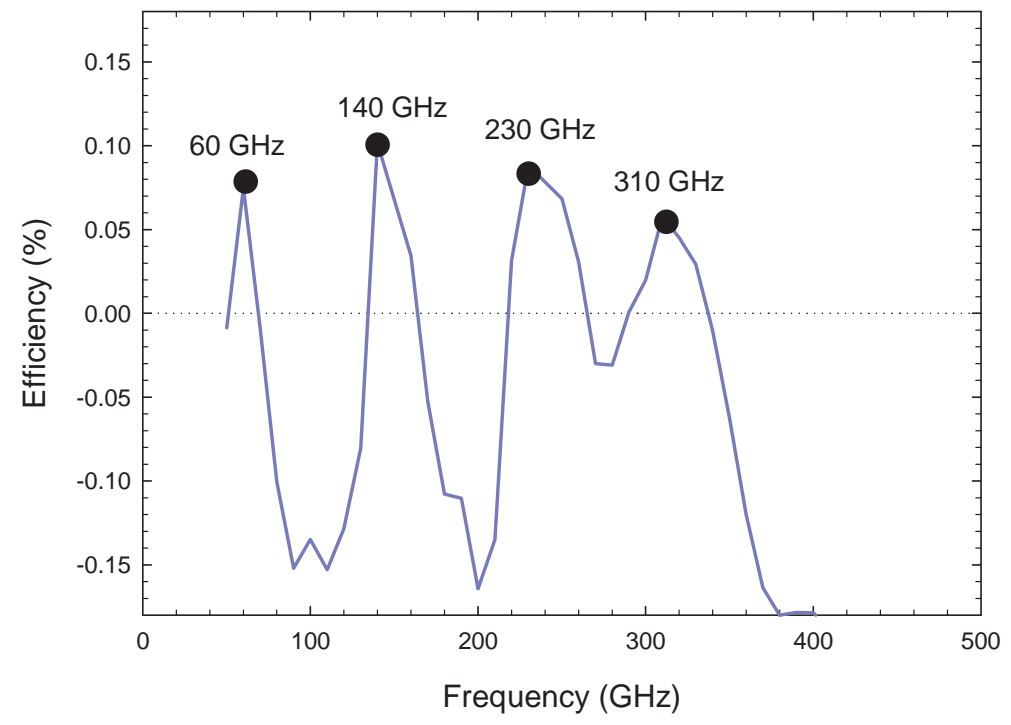

FIG. 5. 

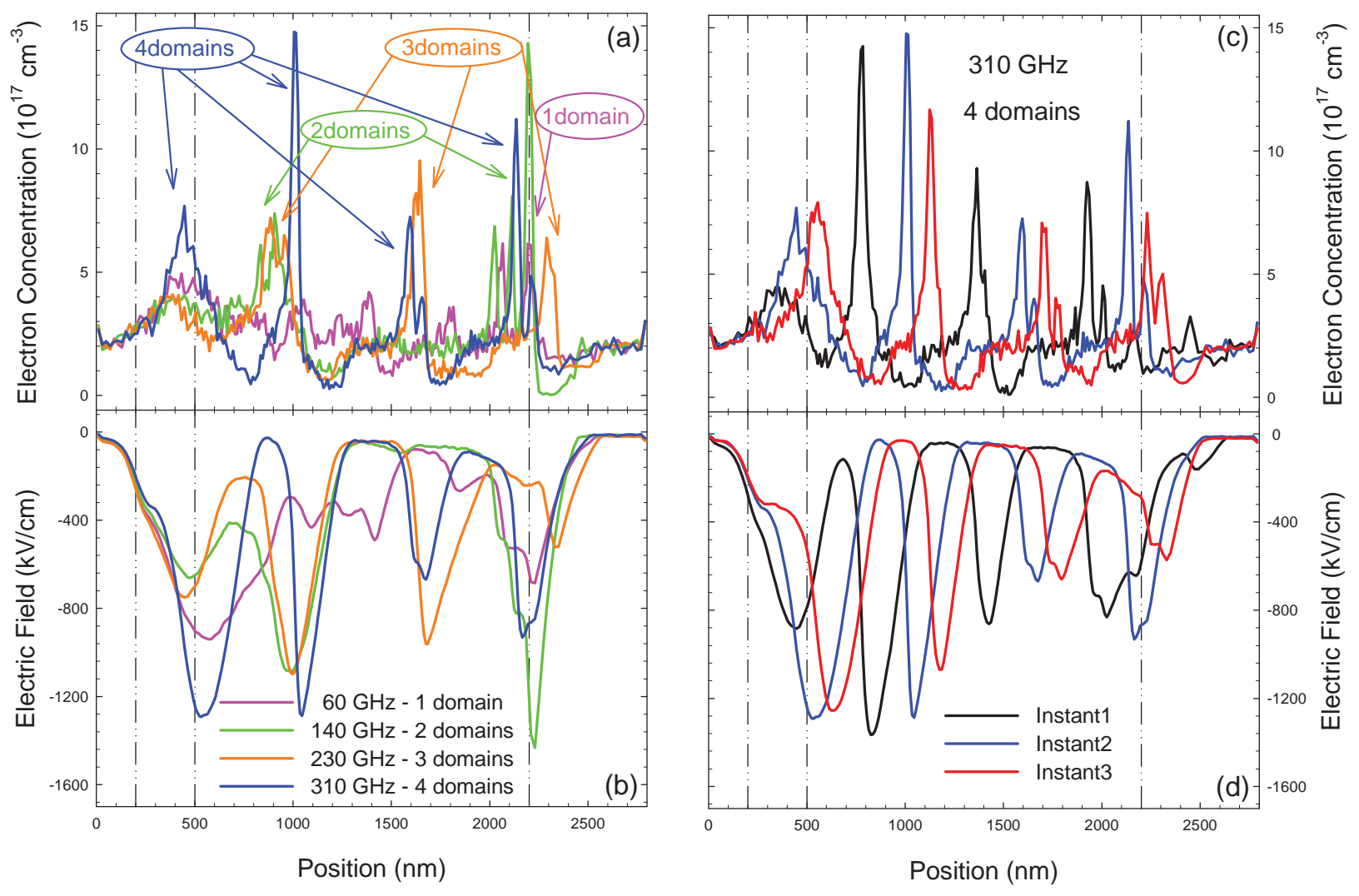

FIG. 6. 

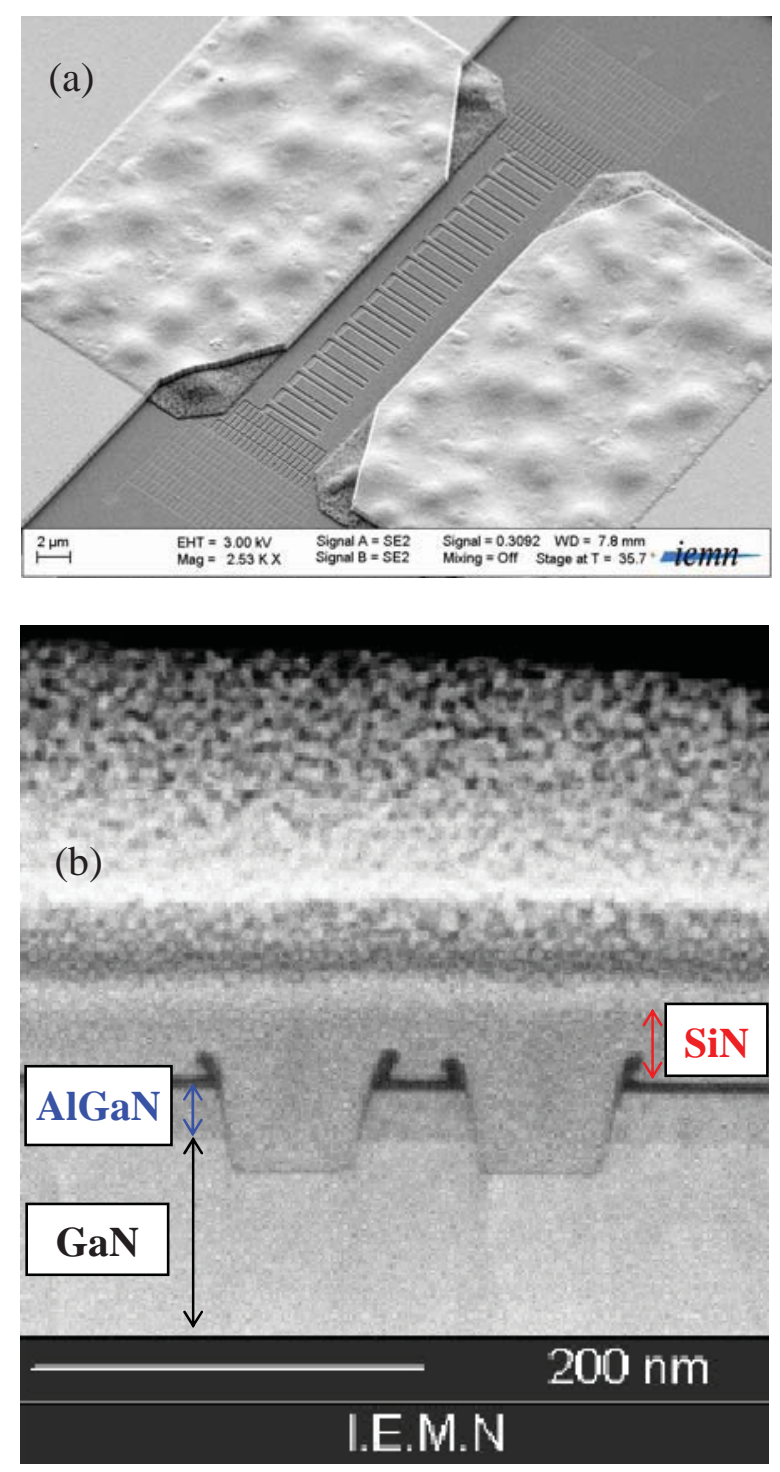

FIG. 7. 


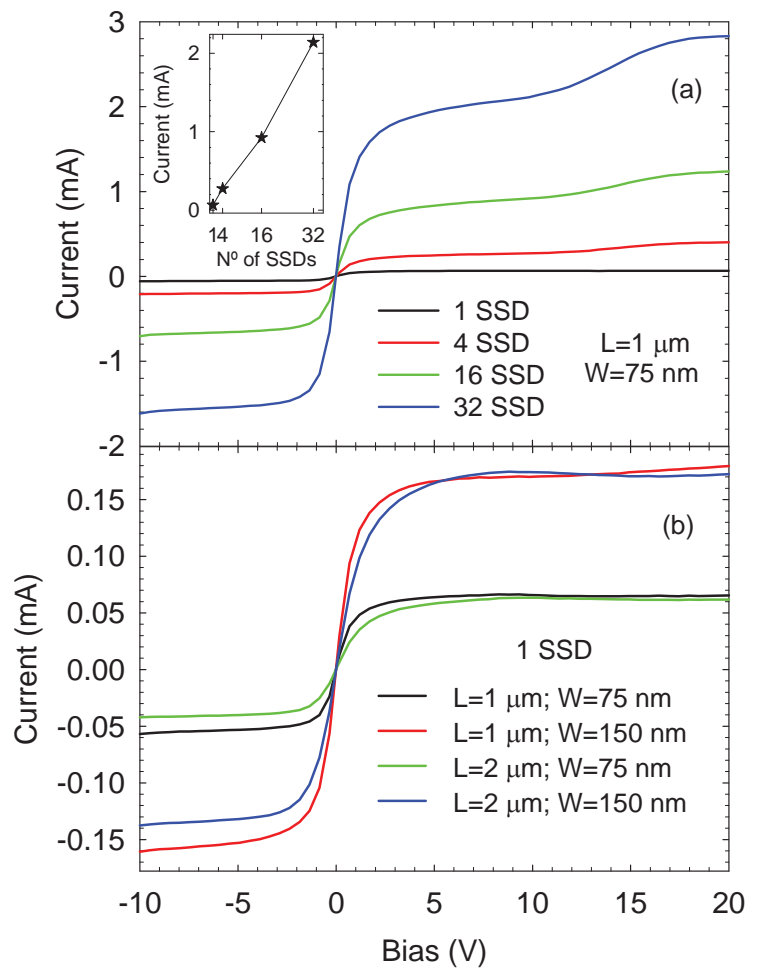

FIG. 8. 


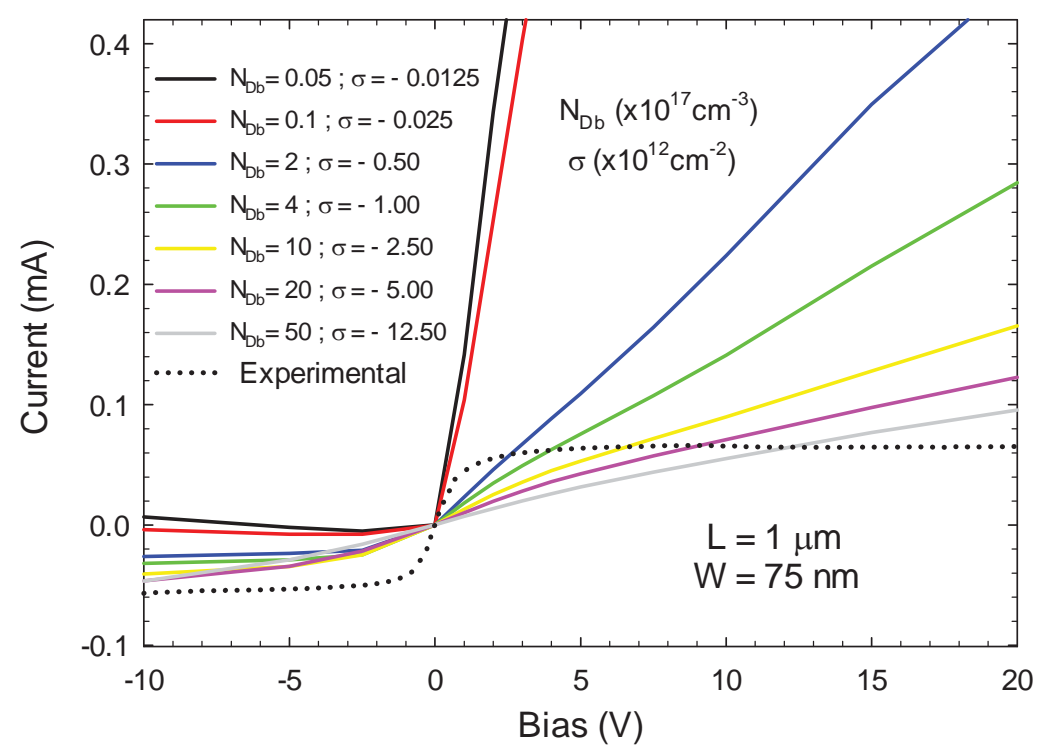

FIG. 9. 

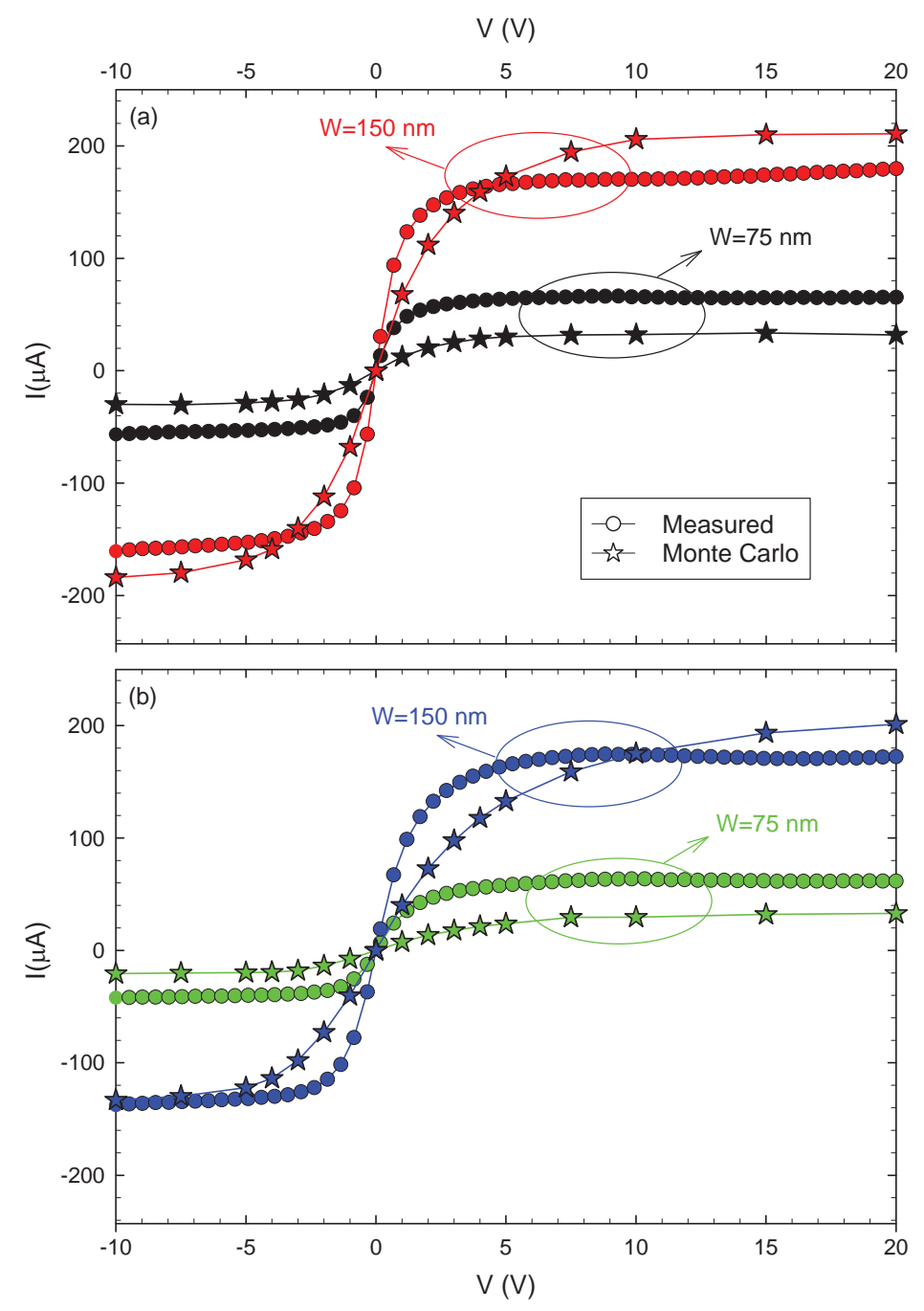

FIG. 10. 


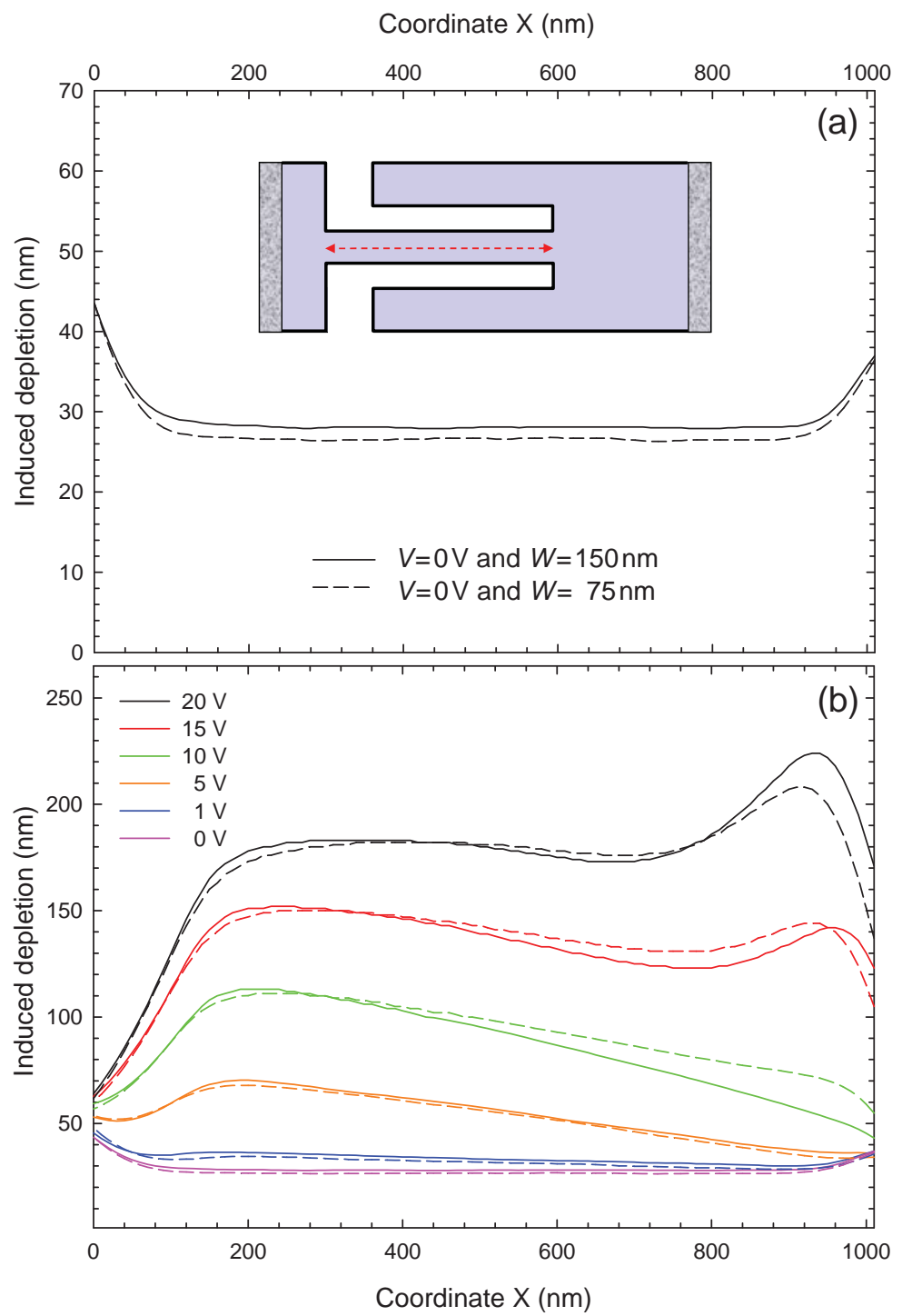

FIG. 11. 


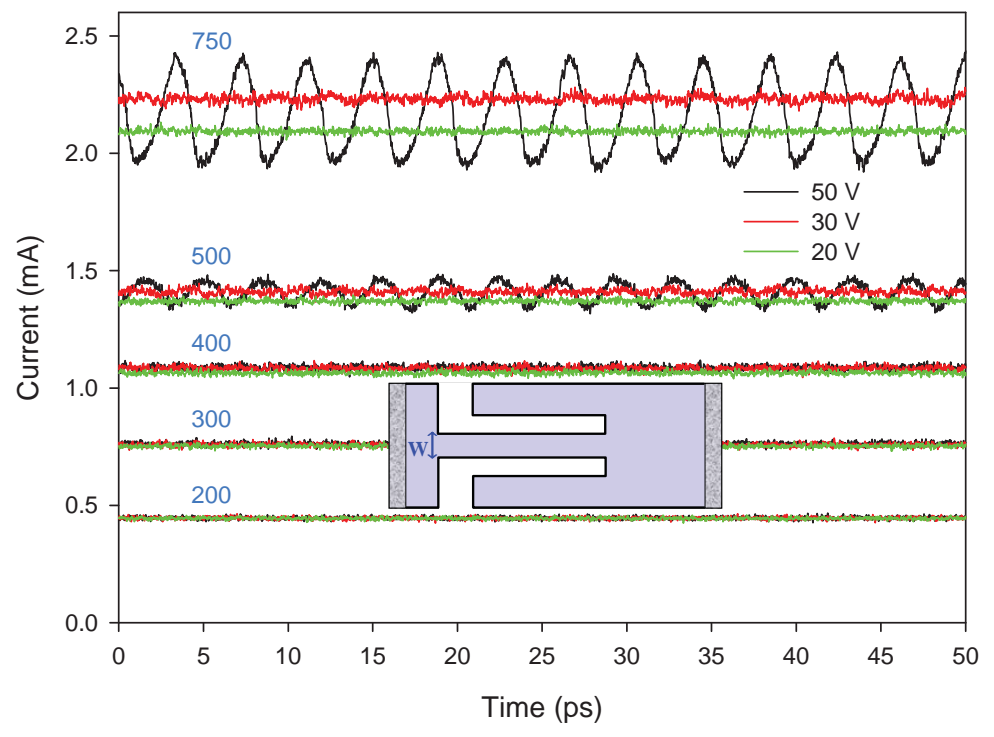

FIG. 12. 